\title{
Polar confinement of the Sun's interior magnetic field by laminar magnetostrophic flow
}

\author{
TOBY S. WOOD† AND MICHAEL E. MCINTYRE \\ Department of Applied Mathematics and Theoretical Physics, University of Cambridge, \\ Cambridge CB3 0WA, UK
}

(Received 28 May 2010; revised 10 December 2010; accepted 18 February 2011; first published online 19 April 2011)

The global-scale interior magnetic field $\boldsymbol{B}_{i}$ needed to account for the Sun's observed differential rotation can be effective only if confined below the convection zone in all latitudes including, most critically, the polar caps. Axisymmetric solutions are obtained to the nonlinear magnetohydrodynamic equations showing that such polar confinement can be brought about by a very weak downwelling flow $U \sim 10^{-5} \mathrm{~cm} \mathrm{~s}^{-1}$ over each pole. Such downwelling is consistent with the helioseismic evidence. All three components of the magnetic field $\boldsymbol{B}$ decay exponentially with altitude across a thin, laminar 'magnetic confinement layer' located at the bottom of the tachocline and permeated by spiralling field lines. With realistic parameter values, the thickness of the confinement layer $\sim 10^{-3}$ of the Sun's radius, the thickness scale being the magnetic advection-diffusion scale $\delta=\eta / U$ where the magnetic (ohmic) diffusivity $\eta \approx 4.1 \times 10^{2} \mathrm{~cm}^{2} \mathrm{~s}^{-1}$. Alongside baroclinic effects and stable thermal stratification, the solutions take into account the stable compositional stratification of the helium settling layer, if present as in today's Sun, and the small diffusivity of helium through hydrogen, $\chi \approx 0.9 \times 10^{1} \mathrm{~cm}^{2} \mathrm{~s}^{-1}$. The small value of $\chi$ relative to $\eta$ produces a double boundary-layer structure in which a 'helium sublayer' of smaller vertical scale $(\chi / \eta)^{1 / 2} \delta$ is sandwiched between the top of the helium settling layer and the rest of the confinement layer. Solutions are obtained using both semi-analytical and purely numerical, finite-difference techniques. The confinement-layer flows are magnetostrophic to excellent approximation. More precisely, the principal force balances are between Lorentz, Coriolis, pressure-gradient and buoyancy forces, with relative accelerations negligible to excellent approximation. Viscous forces are also negligible, even in the helium sublayer where shears are greatest. This is despite the kinematic viscosity being somewhat greater than $\chi$. We discuss how the confinement layers at each pole might fit into a global dynamical picture of the solar tachocline. That picture, in turn, suggests a new insight into the early Sun and into the longstanding enigma of solar lithium depletion.

Key words: MHD and electrohydrodynamics

\section{Introduction}

This paper analyses a new family of laminar magnetostrophic flows that may be important for confining the interior magnetic field $\boldsymbol{B}_{i}$ needed to explain the Sun's

$\dagger$ Present address: Department of Applied Mathematics and Statistics, University of California at Santa Cruz, CA 96064, USA. Email address for correspondence: toswood@ucsc.edu 
differential rotation. As illustrated in figure 1(a), the differential rotation observed within the convection zone goes over into near-solid rotation within the radiative, stably stratified interior, via a thin shear layer called the 'tachocline' much of which is also stably stratified. The need for the interior field $\boldsymbol{B}_{i}$ has been argued elsewhere (McIntyre 1994; Rüdiger \& Kitchatinov 1997; Gough \& McIntyre 1998, hereafter GM98); the main arguments are briefly recalled below. The observational evidence, along with many ideas about the tachocline, is reviewed and further referenced in a recent major compendium, The Solar Tachocline (Hughes, Rosner \& Weiss 2007), and further discussed in the second edition of Mestel's Stellar Magnetism (Mestel 2011).

By a 'confined' $\boldsymbol{B}_{i}$ we mean a field most if not all of whose lines are contained beneath the convection zone, and held there against magnetic (ohmic) diffusion. Such confinement is well known to be necessary in order for the field to help enforce solid rotation in the interior (e.g. Ferraro 1937; Mestel \& Weiss 1987; Charbonneau \& MacGregor 1993; MacGregor \& Charbonneau 1999), and thereby keep the tachocline thin. Confinement against magnetic diffusion requires fluid motion. So, besides magnetic effects, a realistic theory of confinement must take account of Coriolis effects, stable stratification, baroclinicity, and thermal relaxation. Without these effects we cannot correctly describe, for instance, the overall torque balance, which necessarily involves mean meridional circulations (MMCs) as well as Maxwell stresses.

The first attempt at a tachocline theory was that of Spiegel \& Zahn (1992). It included all the above effects except $\boldsymbol{B}_{i}$. Rüdiger \& Kitchatinov (1997) included $\boldsymbol{B}_{i}$ but omitted the other effects. The first attempt to include all of them was that of GM98, in a line of investigation further developed by Garaud \& Garaud (2008). Meanwhile, the dynamical importance of compositional as well as thermal stratification (e.g. Mestel 1953) was suggested for tachocline theories (McIntyre 2007). In particular, the helium settling layer beneath the tachocline is nearly impermeable to MMCs because of the small diffusivity of helium through hydrogen. This near-impermeability of compositionally stratified regions has been called the 'mu-choke' (Mestel \& Moss 1986). The reality of the Sun's helium settling layer is strongly indicated both by standard solar-evolution models and by helioseismology (e.g. Christensen-Dalsgaard, Proffitt \& Thompson 1993; Ciacio, degl'Innocenti \& Ricci 1997; Elliott \& Gough 1999; Christensen-Dalsgaard \& Thompson 2007, also Christensen-Dalsgaard \& Gough 2011, in prep.). As will be seen, this combination of circumstances gives rise to some new and interesting fluid dynamics.

The need for the interior field $\boldsymbol{B}_{i}$ arises from a well-known difficulty with non-magnetic theories. They tend to spread the strong differential rotation of the convection zone down into the radiative interior. Although sometimes disputed, this is a robust and well-understood consequence of thermal relaxation, interacting with Coriolis effects and gyroscopically-pumped MMCs (Haynes et al. 1991; Spiegel \& Zahn 1992; Elliott 1997; McIntyre 2007; Garaud \& Brummell 2008). As shown by Spiegel \& Zahn and confirmed by Elliott, this downward spreading or burrowing would have produced a tachocline far thicker than observed. The accompanying MMC, acting throughout the Sun's lifetime, would also have prevented the helium settling layer from forming.

To counter the burrowing tendency and to allow the interior to rotate solidly, angular momentum has to be transported somehow from the low-latitude tachocline to the high-latitude tachocline. The non-magnetic horizontal eddy viscosity proposed for this purpose by Spiegel \& Zahn is inconsistent with the properties of non-magnetic stratified turbulence known from many studies of the terrestrial atmosphere (McIntyre 1994, 2003, and references). Angular-momentum transport by internal gravity waves 
(a)

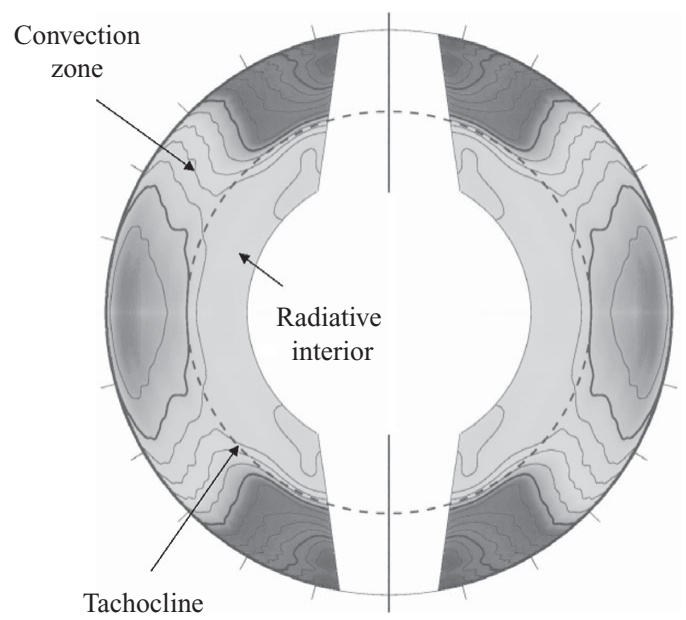

(b)

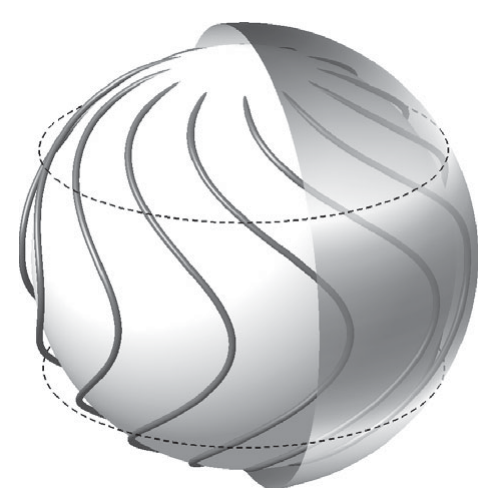

Figure 1. (a) The Sun's differential rotation deduced from helioseismic data using inverse methods (adapted from Schou et al. 1998). The radiative interior rotates approximately solidly with angular velocity $\Omega_{i}=2.7 \times 10^{-6} \mathrm{~s}^{-1}$, or $435 \mathrm{nHz}$. Within the convection zone, the angular velocity increases with colatitude through $350,400,450 \mathrm{nHz}$ (heavy contours) to a maximum just under $470 \mathrm{nHz}$ at the equator. (b) Schematic illustration showing the top of the radiative interior (inner sphere) and the time-averaged magnetic field threading the tachocline just above. The cutaway outer sphere indicates the top of the tachocline, whose depth has been exaggerated. Poloidal magnetic field lines emerge from the interior in high latitudes and are wound up into their curved shapes by the tachocline's differential rotation, acting against turbulent eddy diffusion. A prograde torque is transmitted from low to high latitudes along these field lines. The slow polar and fast equatorial rotation are indicated by the darker shadings of the outer sphere. The dashed lines indicate the latitudes at which the rotation of the convection zone matches that of the interior.

is a physically possible alternative (e.g. Schatzman 1993; Zahn, Talon \& Matias 1997; Rogers \& Glatzmaier 2006; Charbonnel \& Talon 2007, and references). However, it is highly improbable as the main mechanism because, by itself, it has no natural tendency to produce solid rotation at all latitudes and depths (e.g. Plumb \& McEwan 1978).

A suitably-shaped magnetic field can, by contrast, naturally produce the required angular momentum transport, via the Alfvénic elasticity of the field lines. A suitable shape is one in which the field lines link low latitudes to high latitudes within the tachocline. The simplest such shape - simplest by virtue of its axisymmetry - is that suggested schematically in figure $1(b)$, in which the linkage is via a time-averaged field whose lines thread the tachocline, forming the superficial part of a global-scale interior dipole stabilized by a deep toroidal field (e.g. Braithwaite \& Spruit 2004). Such an interior dipole has a diffusive lifetime somewhat greater than the Sun's lifetime of around $4.5 \times 10^{9}$ yr. The dipole imposes an Alfvénic 'Ferraro constraint' on the interior. It is this constraint that helps to enforce the interior's solid rotation (e.g. Ferraro 1937; Mestel \& Weiss 1987; MacGregor \& Charbonneau 1999).

The field lines shown in figure $1(b)$ emerge from the interior (light-grey sphere) near the north pole and, after threading their way through the tachocline, re-enter the interior near the south pole. They return northward through an interior 'applecore' region, not shown, surrounding the rotation axis. It is crucial that the field lines emerging from the interior bend over toward the horizontal as they enter 
the tachocline. They must be prevented from extending upward through the polar cap, as occurs when magnetic diffusion dominates (e.g. Braithwaite \& Spruit 2004; Brun \& Zahn 2006). The curved shapes of the field lines in figure 1(b) are evidently such as to transport angular momentum from low to high latitudes by means of persistent Alfvénic torques, exactly as required to prevent the tachocline's MMCs from burrowing into the interior and thickening the tachocline. This persistent angular momentum transport from the curved field lines might of course be supplemented by transport due to MHD-turbulent stresses within the tachocline (e.g. Spruit 2002; Gilman \& Cally 2007; Parfrey \& Menou 2007).

The time averaging envisaged in figure $1(b)$ conceals a plethora of fast processes, including the 22-year dynamo cycle, convective overshoot, and other turbulent processes arising from instabilities in the tachocline. All these are fast relative to the time scales on which the mean structure of the tachocline is maintained, $\sim 10^{5} \mathrm{yr}$ or more. Apart from possibly contributing to the time-averaged angular momentum transport, the fast processes are presumed to have two important consequences. The first is to produce a turbulent magnetic diffusivity that stops the field lines being wound up arbitrarily tightly by the shear in the tachocline, keeping the curved shapes shown.

The second important consequence is that, away from the poles, the field lines are held down, and held approximately horizontal, by turbulent 'magnetic flux pumping' from the convective overshoot layer. The effectiveness of such flux pumping can be strongly argued from several lines of evidence, including three-dimensional direct numerical simulations, with varying emphasis on the role of turbulent anisotropy and of vertical gradients of density and turbulent intensity (e.g. Tobias et al. 2001; Kitchatinov \& Rüdiger 2008, and references; see also $\S 3$ of Weiss et al. 2004 and $\S 8.6 .1$ of Mestel 2011 for historical reviews).

Near the poles it is less clear that magnetic flux pumping will be effective in confining the field. At least its effectiveness for near-vertical magnetic fields has not, to our knowledge, been convincingly demonstrated. However, as argued for instance in GM98, there are good reasons in any case ( $\$ 2$ below) to expect the tachocline's MMC near the poles to take the form of weak but persistent downwelling. This suggests that the field can, in any case, be confined in the polar caps through an advection-diffusion balance, the kind of balance argued for heuristically in GM98. The purpose of this paper is to show in detail, by solving an appropriate set of nonlinear magnetohydrodynamic equations, that such polar confinement by downwelling is indeed possible in a physically realistic model, applicable to the Sun both today and early in its main-sequence lifetime.

A large family of axisymmetric nonlinear solutions showing polar confinement has been obtained using two different techniques. The first technique is semi-analytical in a sense to be explained, and the second is numerical on a two-dimensional grid. The solutions are to be regarded as candidate solutions for possible flows in the real Sun, all showing confinement in the sense that the total magnetic field strength $|\boldsymbol{B}|$ dies off exponentially with altitude, thanks to downward advection acting against upward diffusion. In this sense the poloidal and toroidal field components are both well confined. We call these flows 'confinement layers'. They are not to be confused with the tachocline itself. Rather, they occupy relatively thin regions at the bottom of the tachocline and are much more weakly sheared, with relatively long time scales.

The detailed dynamics involves not only magnetic advection, stretching, twisting and diffusion but also a near-perfect balance between Lorentz, Coriolis, pressure-gradient and buoyancy forces ( $\S \S 3 \mathrm{ff}$.). Thus the confinement-layer flows are magnetostrophic, 


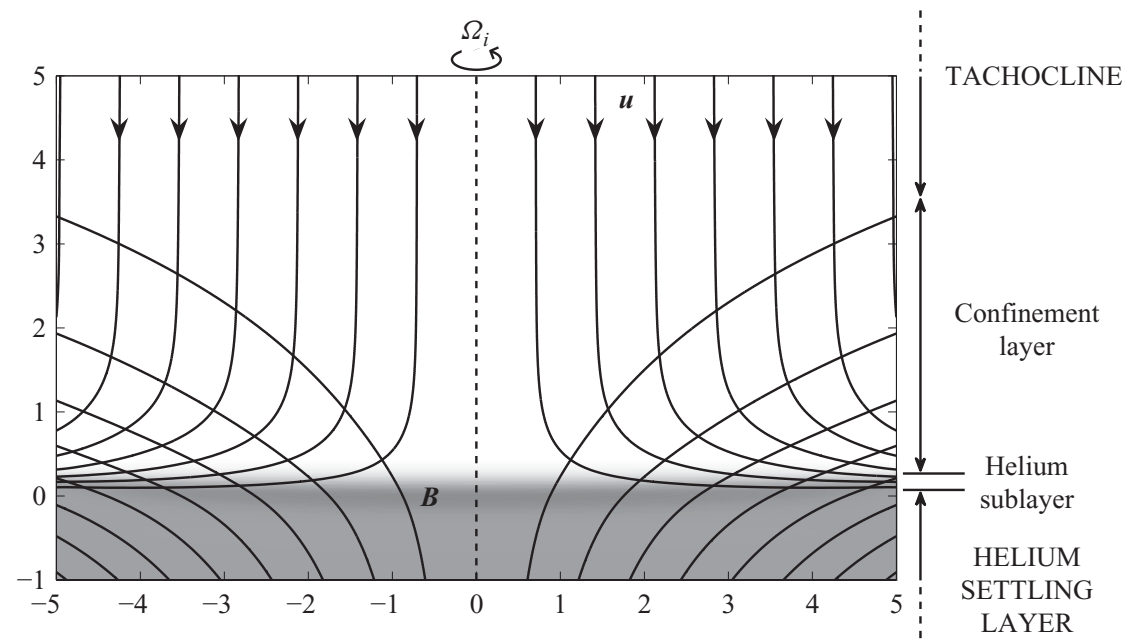

FiguRE 2. The magnetic confinement layer near the north pole in a model for today's Sun. The field strength $|\boldsymbol{B}|$ falls off exponentially with altitude $z$. The toroidal components of $\boldsymbol{B}$ and velocity $\boldsymbol{u}$ are not shown. The streamlines with arrows show the downwelling responsible for the confinement. If the downwelling were switched off, the field near the pole would diffuse and become nearly vertical, as illustrated for instance in Brun \& Zahn (2006). Compositional stratification is indicated by shading. The plot is from a numerical solution; the corresponding semi-analytical solution looks almost identical. The horizontal and vertical axes are colatitudinal distance $r$ and altitude $z$ in units of $\delta$, the advection-diffusion scale defined in (1.1). With typical parameter values, the scale $\delta$ is of the order of a fraction of a megametre, $\sim 10^{-3}$ of the Sun's radius.

like certain flows that have been studied in connection with models of the Earth's liquid core (e.g. Kleeorin et al. 1997, and references therein), though different in most other respects. For instance the latter flows are viscous but unstratified: buoyancy forces and thermal diffusion are absent. In the confinement-layer flows studied here, by contrast, viscosity turns out to be wholly unimportant while buoyancy and thermal diffusion are crucial, along with magnetic diffusion.

Figure 2 gives a preview of a typical confinement-layer flow, seen in vertical cross-section. It shows the poloidal velocity and magnetic field components from a numerical solution. The emerging magnetic field lines are bent over within the confinement layer, as required to fit into the global picture sketched in figure $1(b)$. The magnetic field $\boldsymbol{B}$ has a toroidal component, not shown in the figure, imparting spiral shapes to the three-dimensional field lines and providing the prograde Alfvénic torque demanded by the global picture, in balance with a retrograde Coriolis torque on the equatorward flow.

The vertical and colatitudinal distances in figure 2 are shown in units of the magnetic advection-diffusion scale

$$
\delta=\eta / U
$$

say, where $U$ is the magnitude of the downwelling and $\eta$ is the magnetic diffusivity. Throughout this paper, we assume that the confinement-layer flow is laminar, and therefore use molecular or microscopic diffusivity values $(\S 3)$. Issues of stability or instability lie beyond the scope of this paper but, close to the pole at least, there appears to be a strong case for stability, to be argued in a future paper, arising from the smallness of the scale $\delta$. Under reasonable assumptions, $\delta$ is only a fraction of a 
megametre, far smaller than the thickness of the overlying tachocline which latter, by contrast, is probably unstable and indeed turbulent, as already mentioned (e.g. Spruit 2002; Gilman \& Cally 2007; Parfrey \& Menou 2007).

In the present-day Sun's helium settling layer, the top of which corresponds to the shaded region in figure 2, a downward gradient of helium concentration reinforces the stable stratification due to the sub-adiabatic temperature gradient. Because the diffusivity of helium through hydrogen, $\chi \approx 0.9 \times 10^{1} \mathrm{~cm}^{2} \mathrm{~s}^{-1}$, is much less than the magnetic diffusivity $\eta \approx 4.1 \times 10^{2} \mathrm{~cm}^{2} \mathrm{~s}^{-1}$, the helium settling layer is nearly impermeable to the confinement-layer flow. Helium advection and diffusion are comparable only in the extremely thin 'helium sublayer' marked in figure 2. In this and other respects, all the solutions in the present paper supersede those described in a first report on this work (Wood \& McIntyre 2007, hereafter WM07). For instance, in WM07 we took $\chi$ to be zero, implying a helium sublayer of vanishing thickness. We also took $v$, the kinematic viscosity, to be zero and allowed a finite slip velocity at the top of the helium settling layer, assuming that this slip velocity would in reality be resolved into a weak Ekman layer. However, the solutions presented here show that, on the contrary, no Ekman layer forms. The slip discontinuity is replaced by a smooth velocity profile across the helium sublayer and, as will be shown in $\S 6$, the flow stays essentially inviscid.

The plan of the paper is as follows. In $\S 2$ we summarize the reasons for expecting persistent downwelling over the poles. In $\S 3$ we present the model equations and in $\S 4$ the semi-analytical solutions. Those solutions rely on assuming a selfsimilar horizontal structure that is asymptotically valid in the limit of strong stable stratification. The same limit was taken in WM07.

The validity of the strong-stratification limit is assessed in $\S \S 5$ and 6 , which take a thorough look at the dynamical balances and scalings in the confinement layer and helium sublayer respectively. Strong stable stratification means that the thermal and compositional stratification surfaces are 'flat', meaning gravitationally horizontal, to sufficient approximation in some region surrounding the poles, which for reasonable parameter values can be quite large in horizontal extent, up to tens of degrees of colatitude. Within the helium sublayer, the low magnetic Reynolds number and flat geometry cause the momentum balance to take on the character of flow in a porous medium, as fluid pushes horizontally past the field lines. As already indicated, true viscous effects are negligible everywhere, even in the sublayer.

Boundary conditions for the numerical solutions are discussed in $\S 7$. The numerical solutions themselves are presented and discussed in $\S 8$. They provide cross-checks with the semi-analytical solutions plus additional insights. In particular, they directly demonstrate the flatness of the stratification surfaces by solving the full equations, for finite stratification. The solutions allow the stratification surfaces to tilt as they may, but confirm that the departures from flatness are indeed small when the stratification is realistically strong. In figure 2, for instance, the departures from flatness are barely visible.

In $\S 9$ we discuss a subtlety that arises when comparing the semi-analytical and numerical solutions in the upper part of the flow. The dynamical balances aloft become delicate as the Lorentz and Coriolis forces become vanishingly small. The effects of truncation error and other small effects thus complicate the comparison. However, this is something of an academic point because of our expectation that, in reality, the confinement-layer solutions will need to be matched to a turbulent tachocline aloft, a task that remains a challenge for the future. 
In $\S 10$ we show that the presence of the helium settling layer is not crucial to our confinement-layer model. The interior field $\boldsymbol{B}_{i}$ is sufficient by itself to turn the flow equatorward, and the field remains confined in much the same way. That result has relevance to the Sun's early main-sequence evolution. It explains for instance how the burrowing tendency could have been held in check from the start, allowing the helium settling layer to form. In the concluding discussion, $\S 11$, we consider the implications for early solar evolution and lithium depletion.

\section{Downwelling in the polar tachocline}

Our polar-confinement scenario relies on the MMC pattern in the stably-stratified polar tachocline being robustly and persistently downward above the confinement layer, after averaging out any fast fluctuations due to waves and turbulence. As recognized in GM98, helioseismology provides a compelling reason to expect polar downwelling rather than upwelling, at least in today's tachocline. A further reason is that a downward MMC over the pole is a robust consequence of the gyroscopic pumping already mentioned, which, in the absence of the interior field $\boldsymbol{B}_{i}$, would mediate the downward spreading or burrowing of the convection zone's slow polar rotation. The distinction between gyroscopically-pumped MMCs and MMCs driven in other ways is reviewed in McIntyre (2007, $\S \S 8.1-8.2)$, confirming also the robustness of the burrowing tendency itself, despite recent controversy. The upshot is that we expect persistent polar downwelling to be present not only in today's Sun, but also throughout the Sun's main-sequence lifetime.

The argument from helioseismology is as follows. As is well known, the pressure, density and angular velocity fields, averaged with respect to time and longitude, satisfy hydrostatic and cyclostrophic balance to excellent approximation. Departures from such balance must take the form of fast oscillations such as $p$-modes and $g$-modes, or turbulent fluctuations. From the curl of the momentum equation, taking its azimuthal component, we may show in the standard way that balance implies the so-called 'thermal-wind relation'. In cylindrical polar coordinates $(z, r, \phi)$ centred on the axis of rotation, with the axial coordinate $z$ directed vertically upward at the north pole, the thermal-wind relation can be expressed as

$$
r \rho^{2} \frac{\partial|\boldsymbol{\Omega}|^{2}}{\partial z}=(\nabla p \times \nabla \rho) \cdot \boldsymbol{e}_{\phi},
$$

where $\boldsymbol{\Omega}$ is the absolute angular velocity of the Sun's differential rotation, $\rho$ is the density, and $p$ is the total pressure. The unit vector $\boldsymbol{e}_{\phi}$ is directed azimuthally, while $\nabla p$, being dominated by its hydrostatic part, is very close to being vertically downward. On the assumption that the observed negative sign of $\partial|\boldsymbol{\Omega}|^{2} / \partial z$ persists into the region near the pole invisible to helioseismology - Occam's razor makes this a reasonable assumption - we must have a minimum in $\rho$, and hence a maximum in temperature $T$, on each isobaric surface at the pole.

The stably-stratified radiative envelope is a thermally relaxing system. Local temperature anomalies, defined as departures of $T$ from local radiative equilibrium, will tend to relax back toward zero. To hold $T$ above radiative equilibrium near the pole, there has to be persistent adiabatic compression by downwelling, with compensating upwelling and negative $T$ anomalies in lower latitudes.

The strength $U$ of the polar downwelling is difficult to estimate precisely. Among other things it depends on the tachocline thickness, which is not well constrained by helioseismology. The thickness scale enters both via (2.1) and, more sensitively, via the rate of diffusive thermal relaxation within the tachocline. GM98 estimated 
$U \sim 10^{-5} \mathrm{~cm} \mathrm{~s}^{-1}$, using the rather small tachocline thickness estimate, $13 \mathrm{Mm}$, derived by Elliott \& Gough (1999). The value of $U$ thus estimated is inversely proportional to the cube of the tachocline thickness, and so a similar estimate using a deeper tachocline would yield a much smaller value of $U$.

However, GM98 assumed that the bulk of the tachocline is laminar. McIntyre (2007) considered an alternative scenario in which magnetohydrodynamic turbulent stresses within a deeper tachocline dominate the angular-momentum transport from the overlying convection zone, except near the bottom of the tachocline. The turbulent stresses were estimated by assuming a particular prescription for the turbulence, following Spruit (2002). The stresses diverge in a thin layer near the bottom of the tachocline, just above the confinement layer, where they gyroscopically pump a downwelling of the order of $U \sim 4 \times 10^{-5} \mathrm{~cm} \mathrm{~s}^{-1}$ or greater.

Fortunately, our confinement-layer solutions can accommodate a wide range of uncertainty over the value of $U$. They will show that polar field confinement by downwelling is robust over a range of $U$ values at least as wide as $10^{-6} \mathrm{~cm} \mathrm{~s}^{-1}$ to $10^{-4} \mathrm{~cm} \mathrm{~s}^{-1}$. From here on we use GM98's value $U \sim 10^{-5} \mathrm{~cm} \mathrm{~s}^{-1}$ for illustrative purposes.

\section{The model equations}

Consider the magnetic confinement layer near the north pole. As already noted, the magnetic advection-diffusion thickness scale $\delta=\eta / U$ is to be evaluated with the microscopic magnetic diffusivity $\eta$, whose value in the neighbourhood of the tachocline is carefully estimated by Gough (2007) to be $\eta \approx 4.1 \times 10^{2} \mathrm{~cm}^{2} \mathrm{~s}^{-1}$. This gives the value $\delta \approx 0.4 \mathrm{Mm}$ if $U \approx 10^{-5} \mathrm{~cm} \mathrm{~s}^{-1}$.

We work in a frame rotating with the same angular velocity as the interior, $\Omega_{i}=2.7 \times 10^{-6} \mathrm{~s}^{-1}$, and seek axisymmetric solutions of the Boussinesq MHD equations within a domain consisting of a cylindrical volume $V$ surrounding the pole. Cylindrical coordinates $(z, r, \phi)$ centred on the rotation axis will be used, with corresponding unit vectors $\left(\boldsymbol{e}_{z}, \boldsymbol{e}_{r}, \boldsymbol{e}_{\phi}\right)$. The use of cylindrical coordinates will lead to significant mathematical simplifications. We may regard them as slightly-distorted spherical coordinates, with $r$ representing approximate colatitudinal distance and $z$ locally vertical. The confinement-layer flows to be studied are thin-shell flows, with $z \sim \delta$ and $r \gg \delta$, and so the coordinate distortions should be qualitatively unimportant out to colatitudes as far as $20^{\circ}$ or so.

The Boussinesq framework should itself be highly accurate because typical flow and Alfvén velocities are tiny fractions of the local sound speed, and because $\delta$ values of the order of a fraction of a megametre are far smaller than the pressure scale height, $\approx 60 \mathrm{Mm}$. Conveniently, Boussinesq dynamics permits us to measure the strength of the magnetic field $\boldsymbol{B}$ in terms of the corresponding Alfvén speed, with 1 gauss corresponding to $0.6 \mathrm{~cm} \mathrm{~s}^{-1}$ at a (constant) tachocline density of $0.2 \mathrm{~g} \mathrm{~cm}^{-3}$ (Gough 2007).

We impose uniform downwelling of magnitude $U$ aloft and a simple axial dipolar, fully-diffused poloidal magnetic field structure beneath, to represent the interior magnetic field $\boldsymbol{B}_{i}=\left(B_{i z}, B_{i r}, B_{i \phi}\right)$, on to which the field $\boldsymbol{B}$ in the confinement layer is to be matched. This interior dipolar field has $B_{i r} / r$ constant and $B_{i z}$ a linear function of $z$. It is possible to have $B_{i \phi} \neq 0$ with $B_{i \phi} / r$ constant; however, for reasons to be explained at the end of the section, the main focus will be on the purely poloidal case $B_{i \phi}=0$. This implies the vanishing of the Alfvénic torque beneath. For the semi-analytical solutions, the condition $B_{i \phi}=0$ is imposed directly. For the numerical solutions a less direct procedure is necessary, to be explained in $\S 8$. 
It is convenient to non-dimensionalize the equations using $\delta$ as the length scale in the horizontal $(r)$ as well as in the vertical $(z)$ direction. Thus the thin-shell nature of the flow is expressed by the dimensionless relation $r \gg 1$. We take $U$ as the scale for the velocity field $\boldsymbol{u}$, and $\delta / U$ as the time scale, $\sim 10^{5} \mathrm{yr}$ if $U \sim 10^{-5} \mathrm{~cm} \mathrm{~s}^{-1}$. This is the advection time scale for the flow through the confinement layer and, by construction, is also the time scale on which $\boldsymbol{B}$ diffuses across the confinement layer. Since this time scale far exceeds the typical turnover time of the turbulent eddies in the overlying layers, we may neglect any fluctuations in the downwelling aloft. We therefore take $U$ to be steady as well as uniform, representing the time-averaged downwelling that is gyroscopically pumped by turbulence in the overlying layers, whether those layers consist of the convection zone or the tachocline or both. Fluctuations in $U$ may well be present, but should not greatly influence the structure of the confinement layer on time scales $\sim 10^{5} \mathrm{yr}$ or more. Even if the downwelling comes in pulses, the cumulative effect will arguably be much the same as if it were steady.

We non-dimensionalize the magnetic field $\boldsymbol{B}$ (expressed as Alfvén velocity) with respect to a different velocity scale $\left(2 \Omega_{i} \eta\right)^{1 / 2} \approx 0.05 \mathrm{~cm} \mathrm{~s}^{-1}$. The significance of this last choice will emerge in $\S 5$. It will simplify the scaling relations (5.5)-(5.10). We suppose that the thermal and compositional stratifications are approximately uniform within the helium settling layer, shaded in figure 2, since the confinement layer's MMCs do not penetrate into that region. Writing $\hat{\vartheta}$ and $\hat{\mu}$ for the fractional (therefore dimensionless) perturbations of Boussinesq potential temperature and mean molecular weight, we therefore impose that the corresponding buoyancy frequencies are exactly constant at the bottom of the domain. That is, we impose, with $z$ now the dimensionless vertical coordinate,

$$
\begin{aligned}
& \left.\frac{\partial \hat{\vartheta}}{\partial z}\right|_{b o t t o m}=\frac{N_{\vartheta}^{2} \delta}{g}=\text { const., } \\
& \left.\frac{\partial \hat{\mu}}{\partial z}\right|_{\text {bottom }}=-\frac{N_{\mu}^{2} \delta}{g}=\text { const., }
\end{aligned}
$$

the dimensional buoyancy frequencies $N_{\vartheta}$ and $N_{\mu}$ being constant by definition. For today's Sun we have $N_{\vartheta} \approx 0.8 \times 10^{-3} \mathrm{~s}^{-1}$ (Gough 2007, and D. O. Gough 2010, personal communication), and $N_{\mu} \approx 0.5 \times 10^{-3} \mathrm{~s}^{-1}$ (e.g. Christensen-Dalsgaard \& Thompson 2007), $\dagger$ corresponding to a total buoyancy frequency $N=\left(N_{\vartheta}^{2}+N_{\mu}^{2}\right)^{1 / 2} \approx$ $0.94 \times 10^{-3} \mathrm{~s}^{-1}$ representative of the stratification just inside the helium settling layer. In place of $\hat{\vartheta}$ and $\hat{\mu}$ it proves convenient to define rescaled quantities $\vartheta$ and $\mu$, also dimensionless, by

$$
\begin{aligned}
& \frac{N_{\vartheta}^{2} \delta}{g} \vartheta=\hat{\vartheta}, \\
& \frac{N_{\mu}^{2} \delta}{g} \mu=\hat{\mu},
\end{aligned}
$$

so that the dimensionless stratifications inside the helium settling layer become simply $\partial \vartheta / \partial z=1$ and $\partial \mu / \partial z=-1$.

$\dagger$ Estimates of $N_{\mu}$ vary (e.g. Christensen-Dalsgaard et al. 1993). The value $N_{\mu} \approx 0.5 \times 10^{-3} \mathrm{~s}^{-1}$ was computed in McIntyre $(2007, \S 8.5)$ from information given in Christensen-Dalsgaard \& Thompson (2007). However, the results presented in this paper are not critically dependent on the value of $N_{\mu}$; see $\S 6$. 
Finally, we non-dimensionalize the pressure anomaly $p^{\prime}$ by $2 \Omega_{i} \eta \rho$ where $\rho \approx 0.2 \mathrm{~g} \mathrm{~cm}^{-3}$, the constant Boussinesq density, and thus arrive at the following dimensionless equations:

$$
\begin{aligned}
R o \frac{\mathrm{D} \boldsymbol{u}}{\mathrm{D} t}+\boldsymbol{e}_{z} \times \boldsymbol{u} & =-\nabla p^{\prime}+\alpha_{\vartheta} \vartheta \boldsymbol{e}_{z}-\alpha_{\mu} \mu \boldsymbol{e}_{z}+(\nabla \times \boldsymbol{B}) \times \boldsymbol{B}+E k \nabla^{2} \boldsymbol{u}, \\
0 & =\nabla \cdot \boldsymbol{u}, \\
\frac{\partial \boldsymbol{B}}{\partial t} & =\nabla \times(\boldsymbol{u} \times \boldsymbol{B})+\nabla^{2} \boldsymbol{B}, \\
0 & =\nabla \cdot \boldsymbol{B}, \\
\frac{\mathrm{D} \vartheta}{\mathrm{D} t} & =\frac{\kappa}{\eta} \nabla^{2} \vartheta, \\
\frac{\mathrm{D} \mu}{\mathrm{D} t} & =\frac{\chi}{\eta} \nabla^{2} \mu,
\end{aligned}
$$

where $\mathrm{D} / \mathrm{D} t=\partial / \partial t+\boldsymbol{u} \cdot \nabla$, the material derivative, and where $\boldsymbol{e}_{z}$ is the vertical unit vector. The thermal and compositional diffusivities $\kappa$ and $\chi$ take numerical values $\kappa \approx 1.4 \times 10^{7} \mathrm{~cm}^{2} \mathrm{~s}^{-1}$ and $\chi \approx 0.9 \times 10^{1} \mathrm{~cm}^{2} \mathrm{~s}^{-1}$ in the neighbourhood of the tachocline (Gough 2007). In (3.10) we have neglected gravitational settling, an excellent approximation in virtue of the short time scale $\sim 10^{5} \mathrm{yr}$ of the confinement-layer dynamics relative to the Sun's lifetime, $\gtrsim 10^{9} \mathrm{yr}$. We have defined four dimensionless constants

$$
\begin{gathered}
\alpha_{\vartheta}=\frac{N_{\vartheta}^{2} \delta^{2}}{2 \Omega_{i} \eta}, \quad \alpha_{\mu}=\frac{N_{\mu}^{2} \delta^{2}}{2 \Omega_{i} \eta}, \\
R o=\frac{U}{2 \Omega_{i} \delta}=\frac{\eta}{2 \Omega_{i} \delta^{2}}, \quad \text { and } \quad E k=\frac{v}{2 \Omega_{i} \delta^{2}}=\frac{v}{\eta} R o
\end{gathered}
$$

where $v$ is the kinematic viscosity, the sum of molecular and radiative contributions, $\approx 2.7 \times 10^{1} \mathrm{~cm}^{2} \mathrm{~s}^{-1}$ in the neighbourhood of the tachocline (Gough 2007). The Rossby and Ekman numbers, $R o$ and $E k$, quantify how far magnetic flux and fluid momentum diffuse across the confinement layer during one solar rotation. For $U \sim 10^{-5} \mathrm{~cm} \mathrm{~s}^{-1}$ the Rossby number is tiny, $R o \sim 0.5 \times 10^{-7}$, and the Ekman number is smaller still because $v / \eta \approx 0.7 \times 10^{-1}$. To excellent approximation, therefore, the flows under consideration will be magnetostrophic. That is, in (3.5) the Coriolis force will be balanced against the combined pressure-gradient, buoyancy, and Lorentz forces:

$$
\boldsymbol{e}_{z} \times \boldsymbol{u}=-\nabla p^{\prime}+\alpha_{\vartheta} \vartheta \boldsymbol{e}_{z}-\alpha_{\mu} \mu \boldsymbol{e}_{z}+(\nabla \times \boldsymbol{B}) \times \boldsymbol{B},
$$

and this will be verified independently from the numerical solutions, from which magnetostrophic balance emerges rather than being imposed.

We are concerned here only with axisymmetric steady states. Then the azimuthal components of (3.13) and its curl are respectively

$$
\begin{aligned}
u_{r} & =\frac{1}{r} \boldsymbol{B} \cdot \nabla\left(r B_{\phi}\right), \\
\frac{\partial u_{\phi}}{\partial z} & =\alpha_{\vartheta} \frac{\partial \vartheta}{\partial r}-\alpha_{\mu} \frac{\partial \mu}{\partial r}+\frac{1}{r} \frac{\partial}{\partial z}\left(B_{\phi}^{2}\right)-r \boldsymbol{B} \cdot \nabla\left(\frac{[\nabla \times \boldsymbol{B}]_{\phi}}{r}\right),
\end{aligned}
$$

where $r$ is the dimensionless perpendicular distance from the rotation axis, and where suffixes $z, r, \phi$ denote vector components. 
Equation (3.14) represents the local torque balance about the rotation axis, after multiplication by $r$. It describes how the retrograde Coriolis torque from the equatorward flow is balanced by the prograde Lorentz torque from the confined magnetic field. $\dagger$ Equation (3.15) represents thermal-wind balance generalized to include compositional gradients and the Lorentz force-curl. In the upper part of figure 2, where the magnetic field and compositional stratification are both negligible, this balance becomes the standard thermal-wind balance

$$
\frac{\partial u_{\phi}}{\partial z}=\alpha_{\vartheta} \frac{\partial \vartheta}{\partial r}
$$

which is the Boussinesq, low-Rossby-number limit of (2.1) in dimensionless units.

The overall torque balance for the confinement layer can be expressed by integrating $r$ times (3.14) over the volume $V$ of the cylindrical domain, then using the divergence theorem and the fact that $\nabla \cdot \boldsymbol{u}=0$. The result is

$$
\int_{\partial V} \frac{1}{2} r^{2} \boldsymbol{u} \cdot \mathrm{d} \boldsymbol{S}=\int_{\partial V} r B_{\phi} \boldsymbol{B} \cdot \mathrm{d} \boldsymbol{S},
$$

where $\mathrm{d} \boldsymbol{S}$ is the vector area element directed outward. In the corresponding dimensional equation, $\frac{1}{2} r^{2}$ is replaced by $\Omega_{i} r^{2}$, the absolute angular momentum per unit mass after neglecting contributions $O(R o)$.

The right-hand side of (3.17) represents the total Alfvénic torque exerted on the confinement layer. The left-hand side represents the net rate of absolute angular momentum export by the flow coming in through the top and out through the periphery, as in figure 2. For any such velocity field the left-hand side is positive. Therefore the Alfvénic torque must also be positive, i.e. prograde.

The need for the field $\boldsymbol{B}_{i}$ is now apparent. Without it, the balance described by (3.17) would be impossible. Instead, the fluid within $V$, and outside it as well, would begin to be spun down by the flow. In the Sun, this would cause the slow rotation of the high-latitude convection zone to spread down into the radiative envelope, in the Haynes-Spiegel-Zahn burrowing process noted in $\S 1$. We also note that the torque balance described by (3.17) is very different from the torque balance described by Rüdiger \& Kitchatinov (1997) and Kitchatinov \& Rüdiger (2006) in which there is no Coriolis effect, the Lorentz torque being balanced instead by a viscous torque. In fact for realistic solar parameter values it will be seen that, as already indicated, viscous torques are entirely negligible - perhaps counterintuitively for shear layers as thin as the confinement layer and the helium sublayer.

The balance expressed by (3.17) must apply also throughout the interior apple-core region that surrounds the rotation axis, magnetically linking the confinement layer at the north pole to that at the south. The mu-choke, both in the Sun's helium-rich inner core and in the helium settling layer, if present, is enough to suppress MMCs in the apple-core region and make the left-hand side of (3.17) negligible, when the volume of integration $V$ is taken as the interior apple-core region. Any Alfvénic torque exerted on the bottom of one confinement layer must therefore be balanced by an equal and opposite Alfvénic torque exerted on the bottom of the other confinement layer. If we assume that the two confinement layers are mirror-symmetric about the equatorial

$\dagger$ A referee reminds us that this torque balance is related to the well-known Taylor constraint for magnetostrophic flow (Taylor 1963). If (3.14) is integrated vertically between two hypothetical impermeable boundaries then, because $\nabla \cdot \boldsymbol{u}=0$, the integral on the left and hence that on the right must vanish. In the confinement-layer problem, however, this constraint is broken by the presence of downwelling aloft. 
plane, then it follows that the torque, and therefore $B_{i \phi}$, must vanish on the bottom of each confinement layer. The prograde Alfvénic torque on each confinement layer must therefore come wholly from its sideways connection to lower latitudes via the tachocline, which is consistent with the global picture suggested in figure $1(b)$.

\section{The semi-analytical solutions}

Equation (3.15) describes how the Coriolis and Lorentz force-curls act to tilt the stratification surfaces within the confinement layer. Now if the stable stratification is sufficiently strong, then the tilting will be only slight. The formal asymptotic limit to describe this is $\alpha_{\vartheta}, \alpha_{\mu} \rightarrow \infty$ with $\boldsymbol{u}$ and $\boldsymbol{B}$ finite so as to preserve the steady-state torque balance (3.17) and a steady-state balance in the induction equation (3.7). In that limit, (3.15) implies that $\partial \vartheta / \partial r \rightarrow 0$ and $\partial \mu / \partial r \rightarrow 0$, with both sides of (3.15) finite. The stratification surfaces become perfectly flat: $\vartheta \rightarrow \vartheta(z)$ and, in the helium sublayer, $\mu \rightarrow \mu(z)$.

In the limit of perfect flatness thus enforced by (3.15), the remaining equations can be reduced to a system of coupled ordinary differential equations. With $\vartheta=\vartheta(z)$ and $\mu=\mu(z),(3.9)$ and (3.10) imply for steady flow that

$$
u_{z}=\frac{\kappa}{\eta} \frac{\mathrm{d}}{\mathrm{d} z} \ln \frac{\mathrm{d} \vartheta}{\mathrm{d} z}=\frac{\chi}{\eta} \frac{\mathrm{d}}{\mathrm{d} z} \ln \frac{\mathrm{d} \mu}{\mathrm{d} z} .
$$

Therefore $u_{z}$ is also a function of $z$ alone, $u_{z}=u_{z}(z)$. From (3.6) it then follows that $u_{r}$ is $r$ times a function of $z$ alone, on the assumption of regularity at the pole $r=0$. We say that the poloidal velocity field is 'horizontally self-similar'. The induction equation (3.7) then permits a steady poloidal magnetic field that is horizontally self-similar in the same sense. The resulting equations are

$$
\begin{aligned}
r u_{r} & =\boldsymbol{B} \cdot \nabla\left(r B_{\phi}\right), \\
0 & =\frac{1}{r} \frac{\partial\left(r u_{r}\right)}{\partial r}+\frac{\mathrm{d} u_{z}}{\mathrm{~d} z}, \\
r \boldsymbol{u} \cdot \nabla \frac{B_{\phi}}{r} & =r \boldsymbol{B} \cdot \nabla \frac{u_{\phi}}{r}+\left(\nabla^{2}-\frac{1}{r^{2}}\right) B_{\phi}, \\
u_{z} \frac{\mathrm{d} B_{z}}{\mathrm{~d} z} & =B_{z} \frac{\mathrm{d} u_{z}}{\mathrm{~d} z}+\frac{\mathrm{d}^{2} B_{z}}{\mathrm{~d} z^{2}}, \\
0 & =\frac{1}{r} \frac{\partial\left(r B_{r}\right)}{\partial r}+\frac{\mathrm{d} B_{z}}{\mathrm{~d} z} .
\end{aligned}
$$

The azimuthal component of (3.5) is replaced by (3.14) and multiplied by $r$ to give (4.2), expressing the balance between Coriolis and Lorentz torques as before. Equations (4.3)-(4.6) correspond to (3.6)-(3.8); (3.9) and (3.10) have no further role, beyond their connection to the downwelling expressed by (4.1). Equation (4.4) permits more general toroidal magnetic and differential-rotation fields than were considered in WM07.

For large but finite $\alpha_{\vartheta}$ and $\alpha_{\mu}$, departures from perfect flatness arise as small corrections. To describe these corrections it is necessary to bring back (3.9), (3.10) and (3.15); the order of magnitude of the corrections is analysed in $\$ \S 5$ and 6 . The whole picture will be independently checked by the numerical solutions, which automatically contain the departures from flatness governed by (3.9), (3.10) and (3.15) since the full 
set of equations is used, with finite values of $\alpha_{\vartheta}$ and $\alpha_{\mu}$, for instance to produce the almost-flat numerical solution plotted in figure 2 .

Returning now to the limit of perfect flatness, we focus on (4.2)-(4.6). These equations admit a family of solutions in which the function $u_{z}(z)$ is arbitrary except for certain restrictions on its asymptotic behaviour as $z \rightarrow \pm \infty$. In particular, we require $u_{z}(z) \rightarrow-1$ as $z \rightarrow+\infty$ and $u_{z}(z) \rightarrow 0$ as $z \rightarrow-\infty$. These statements will shortly be made more precise. As discussed in $\S 7$, the arbitrariness in $u_{z}(z)$ is needed to permit matching to the confinement layer's surroundings.

Solutions can be most conveniently constructed by taking advantage of the arbitrariness to specify a suitable $u_{z}(z)$ at the start. With $u_{z}(z)$ specified, we can then find $B_{z}(z)$ numerically by solving the vertical component (4.5) of the induction equation as a linear ordinary differential equation, assuming that the time-averaged field $B_{z}$ vanishes far above the confinement layer $(z \rightarrow+\infty)$ and that it matches on to the imposed interior dipolar magnetic field structure beneath $(z \rightarrow-\infty)$. The interior dipole has the same horizontally self-similar structure as the confinement layer, with components satisfying $B_{i r} / r=$ constant, and $B_{i z}$ a linear function of $z$ consistent with (4.6). Even though the balance in (4.5) is not simple advective-diffusive, we find that $B_{z}$ decays upward like $\exp (-z)$.

The radial components of $\boldsymbol{u}$ and $\boldsymbol{B}$ can be found directly from their vertical components, by using (4.3) and (4.6) and assuming regularity at the pole $r=0$ :

$$
\begin{aligned}
& u_{r}=-\frac{r}{2} \frac{\mathrm{d} u_{z}}{\mathrm{~d} z}, \\
& B_{r}=-\frac{r}{2} \frac{\mathrm{d} B_{z}}{\mathrm{~d} z} .
\end{aligned}
$$

So once we have $B_{z}(z)$ we can calculate $B_{r}$ from (4.8), and then the toroidal field $B_{\phi}$ from (4.2) by using (4.7) and taking advantage of the hyperbolic character of the operator $\boldsymbol{B} \cdot \nabla$. By calculating $B_{\phi}$ in this way, we ensure that the Lorentz torque balances the Coriolis torque along each magnetic field line. Requiring that $B_{\phi}(r, z) \rightarrow 0$ for all $r$ as $z \rightarrow-\infty$ (recall the end of $\S 3$ ) leads to the following, unique solution of (4.2):

$$
B_{\phi}=B_{z} \int_{-\infty}^{z} \frac{u_{r}}{B_{z}^{2}} \mathrm{~d} z .
$$

For any $u_{r}$ profile that decays exponentially as $z \rightarrow-\infty$, this solution for $B_{\phi}$, and with it the Maxwell stress and Alfvénic torque, will also decay exponentially as $z \rightarrow-\infty$. The expression (4.9) then shows that $B_{\phi}$ has the same horizontally self-similar functional form as $u_{r}$ and $B_{r}$, namely $r$ times a function of $z$ alone.

To ensure that $\boldsymbol{B}_{\phi}$ decays aloft, as $z \rightarrow+\infty$, it is sufficient to assume that

$$
u_{r}=O(\exp (-\gamma z)) \text { as } z \rightarrow+\infty
$$

for constant $\gamma>1$, implying that $u_{z}(z) \sim-1+O(\exp (-\gamma z))$. The assumption that $\gamma>1$ ensures that we get solutions with qualitatively reasonable behaviour aloft. Otherwise we leave the value of $\gamma$ arbitrary. Once again this is an arbitrariness whose resolution will depend on matching to the confinement layer's surroundings, in this case to conditions aloft. As already indicated, the conditions aloft probably involve turbulent flow, for which we do not yet have quantitative models. So here we restrict ourselves to surveying the possible range of behaviours for $\gamma>1$.

The three cases $\gamma>2, \gamma=2$, and $2>\gamma>1$ need separate consideration. When $\gamma>2$, the only case considered in WM07, the integral in (4.9) converges to a constant 
plus $O(\exp (-(\gamma-2) z))$ as $z \rightarrow+\infty$. That in turn means that $B_{\phi}$ decays upward like $\exp (-z)$. When $\gamma=2$, the integral in (4.9) asymptotes to a linear function of $z$, and $B_{\phi}$ decays upward like $z \exp (-z)$. When $2>\gamma>1$, the integral in (4.9) increases upward like $\exp ((2-\gamma) z)$, but $B_{\phi}$ still decays upward, like $\exp (-(\gamma-1) z)$.

In all these cases it is clear from (4.9) that $B_{\phi}$ does not have exactly the same $z$ dependence aloft as does $B_{z}$. This is contrary to what might have been expected from a naive appeal to advective-diffusive balance, with advection by constant downwelling $u_{z}=-1$. Having the same $z$-dependences would make the right-hand side of (4.2) vanish. Hence it is the more or less subtle departures from advective-diffusive balance, including the contribution to $B_{\phi}$ from the twisting of field lines by the differential rotation $u_{\phi}$, that allow the right-hand side of (4.2) not to vanish and thereby to provide a Lorentz torque to support the flow $u_{r}$ at all altitudes.

The $u_{\phi}$ field that does the twisting can be calculated next, from (4.4) and the condition that the interior rotates solidly, $u_{\phi} \rightarrow 0$ as $z \rightarrow-\infty$. Again this calculation depends on the hyperbolic character of $\boldsymbol{B} \cdot \nabla$. When $B_{\phi}$ is given by (4.9) we have, uniquely,

$$
u_{\phi}=\int_{-\infty}^{z}\left(u_{z} \frac{\partial B_{\phi}}{\partial z}-\frac{\partial^{2} B_{\phi}}{\partial z^{2}}\right) \frac{\mathrm{d} z}{B_{z}}
$$

showing that $u_{\phi}$ is also $r$ times a function of $z$ alone. That is, the differential rotation is what astrophysicists call 'shellular solid rotation'. In the three cases $\gamma>2, \gamma=2$, and $2>\gamma>1$, the behaviours of $u_{\phi}$ as $z \rightarrow+\infty$ are respectively $u_{\phi} \sim$ constant, $u_{\phi} \sim \pm z$, and $u_{\phi} \sim \pm \exp ((2-\gamma) z)$.

Cases with negative shear aloft, $\partial u_{\phi} / \partial z<0$ - especially the last case, with exponentially-increasing negative shear aloft - are suggestive of a possible way to match upward to the observed, much stronger negative shear in the bulk of the tachocline. By using (4.9) to eliminate $B_{\phi}$ from $\partial / \partial z$ of (4.11), then (4.5) to eliminate $\mathrm{d}^{2} B_{z} / \mathrm{d} z^{2}$ and (4.7) to eliminate $\mathrm{d} u_{z} / \mathrm{d} z$, we find

$$
\frac{\partial u_{\phi}}{\partial z}=\frac{u_{z} u_{r}}{B_{z}^{2}}-\frac{1}{B_{z}^{2}} \frac{\partial u_{r}}{\partial z}-\frac{2 u_{r}}{r} \int_{-\infty}^{z} \frac{u_{r}}{B_{z}^{2}} \mathrm{~d} z \approx(\gamma-1) \frac{u_{r}}{B_{z}^{2}} \quad \text { as } z \rightarrow \infty .
$$

The asymptotic behaviour on the right comes from the first two terms in the exact expression. The third term involving the integral is smaller by a factor $O(\exp (-\gamma z))$. Asymptotically, therefore, the sign of the shear $\partial u_{\phi} / \partial z$ aloft is the same as the sign of $u_{r}$ aloft. We can therefore find solutions that match on to the strong negative tachocline shear provided that there is an exponentially weak poleward mass flux above the confinement layer. However, a more precise description of such matching must await future work, for reasons already mentioned. We do not yet have quantitative models of the precise conditions aloft, which are likely to be affected by small-scale MHD turbulence (e.g. Spruit 2002; Gilman \& Cally 2007; Parfrey \& Menou 2007, and references). Aspects of this are touched on again in $\S 9$, the main point for present purposes being that the structure aloft is sensitive to conditions aloft whereas, as is clear from (4.9) and (4.11), the rest of the confinement layer is insensitive to conditions aloft, as we have verified by varying $\gamma$.

Purely for illustration we show one of the solutions in figure 3, somewhat arbitrarily choosing $\gamma=2.24$. In this case the interior field $\boldsymbol{B}_{i}$ is taken such that $B_{r} / r=1$. The downwelling profile $u_{z}(z)$ was adapted from the numerical solution shown in figure 2 , in the manner described in $\S 9$.

Some three-dimensional streamlines and magnetic field lines corresponding to the solution in figure 3 are plotted in figure 4, visualizing how the prograde Lorentz torque 

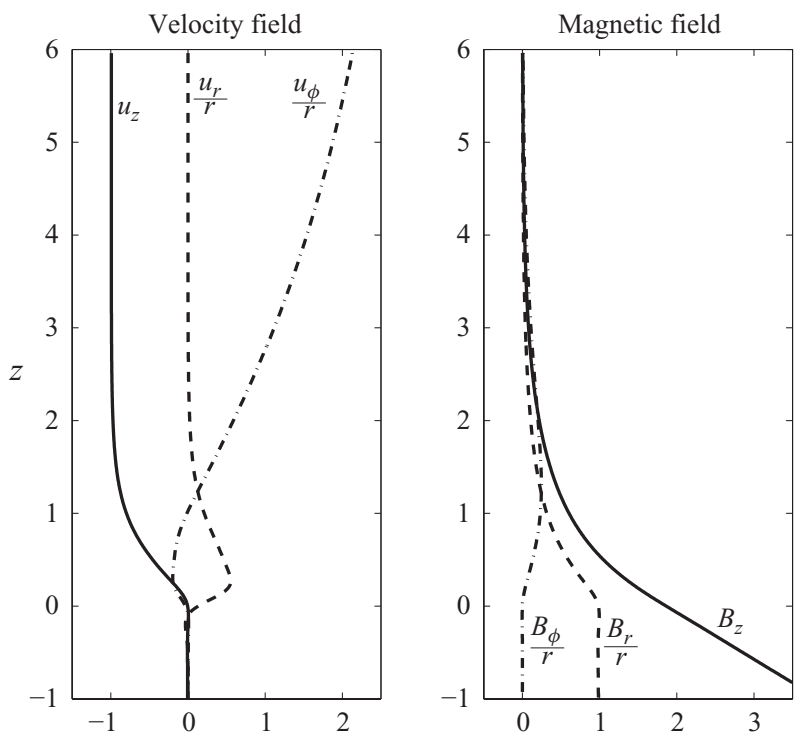

FIGURE 3. Vertical profiles from a semi-analytical solution of the confinement-layer equations in the strong-stratification limit $\alpha_{\vartheta}, \alpha_{\mu} \rightarrow \infty$. The downwelling profile $u_{z}(z)$, solid curve on the left, was chosen to match the downwelling profile from the numerical solution shown in figure 2. For numerical reasons, small adjustments were made to this profile in the 'slippery' upper region $z>1.5 \delta$; see $\S \S 7-9$. In (4.10) the decay constant $\gamma=2.24$, and the $u_{\phi}$ profile therefore approaches a constant like $\exp (-0.24 z)$. The parameter $\Lambda,(5.4)$ below, takes the value $\Lambda \approx 3.5$.

(a)

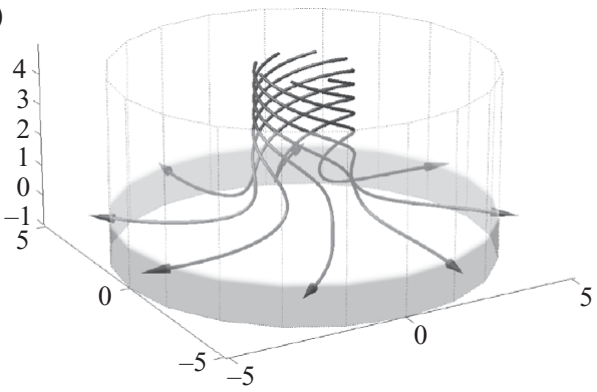

(b)

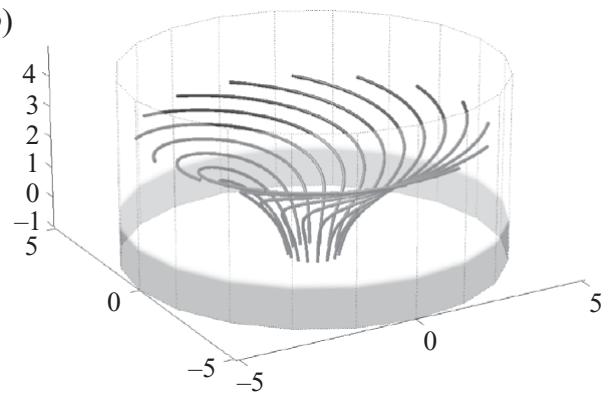

FiguRE 4. Streamlines $(a)$ and magnetic field lines $(b)$ from the semi-analytical solution whose vertical profiles are displayed in figure 3 . The peripheral shading locates the helium settling layer and helium sublayer.

on the right of (4.2), associated with field-line curvature, balances the retrograde Coriolis torque on the left of (4.2) and satisfies the overall torque balance (3.17).

\section{Confinement-layer scalings}

We are interested in the departures from perfect flatness associated with large but finite stratification. We may regard those departures as the $O(\epsilon)$ correction terms in an asymptotic expansion whose leading, $O(1)$ term is a semi-analytical solution, where the small parameter $\epsilon$ is inversely proportional to the stratification and where the limit $\epsilon \rightarrow 0$ is taken within a cylindrical domain of fixed dimensionless size $r=r_{d}$. 
It will be found that the departures from flatness behave like $O\left(\epsilon r^{2}\right)$ near the pole. So instead of saying that the departures are small within the fixed domain $r \leqslant r_{d}$ we may say, perhaps more usefully, that the semi-analytical solutions are valid as leading-order approximations as long as we are well within some dimensionless colatitudinal distance of the pole, $r_{\vartheta}$ say, that is large in comparison with unity. For a fair range of parameter values the dimensional counterpart of $r_{\vartheta}$ turns out to be quite large numerically, of the order of hundreds of megametres, or tens of degrees of colatitude. Of course for the solutions to apply we must also be within a region of approximately uniform downwelling. In this section we use scaling arguments to arrive at an appropriate definition of $r_{\vartheta}$ for the bulk of the confinement layer, where the stratification is purely thermal. The next section presents the corresponding analysis for the helium sublayer.

Consider, then, the scaling regime in the bulk of the confinement layer. Because the photon mean free path makes the thermal diffusivity $\kappa$ relatively large, with $\kappa / \eta \sim 3 \times 10^{4}$, the confinement-layer flow only weakly perturbs the background thermal stratification. Consistently with (3.1) and (3.3) we define a dimensionless thermal anomaly $\vartheta^{\prime}$ such that $\vartheta=z+\vartheta^{\prime}+$ constant, and such that $\vartheta^{\prime} \rightarrow 0$ beneath the confinement layer at the pole. Then, at the pole, and by implication sufficiently close to it, the leading-order balance in the dimensionless steady-state thermal equation (3.9) involves only the vertical component $u_{z}$ of $\boldsymbol{u}$,

$$
u_{z}=\frac{\kappa}{\eta} \nabla^{2} \vartheta^{\prime} .
$$

On the right-hand side we have $\nabla^{2} \vartheta^{\prime} \sim \partial^{2} \vartheta^{\prime} / \partial z^{2} \sim \vartheta^{\prime}$ since in the confinement layer $\partial / \partial z \sim 1$, and since $\vartheta^{\prime} \rightarrow 0$ beneath. Hence with $\left|u_{z}\right| \sim 1$ we have

$$
\vartheta^{\prime} \sim \eta / \kappa \approx 3 \times 10^{-5} \ll 1 .
$$

In the bulk of the confinement layer we may estimate the departure from flatness, equivalently the $r$-dependence of $\vartheta^{\prime}$, from (3.15) with $\alpha_{\mu}$ neglected and $\alpha_{\vartheta}$ considered large. Since the leading-order solution is a semi-analytical solution, the remaining terms in (3.15) all take the form $r$ times a function of $z$ alone, to leading order, from which we may deduce that $\partial \vartheta^{\prime} / \partial r$ is $r$ times a function of $z$ alone and hence that

$$
\vartheta^{\prime}=a_{\vartheta}+b_{\vartheta} r^{2},
$$

to leading order, where $a_{\vartheta}$ and $b_{\vartheta}$ are functions of $z$ alone and where $a_{\vartheta}$ has the small magnitude given by (5.2), $a_{\vartheta} \sim \eta / \kappa$. With $\partial / \partial z \sim 1$ the condition for (5.3) to be compatible with uniform downwelling, $u_{z}=u_{z}(z)$ in (5.1), can now be seen to be $r \ll\left|a_{\vartheta} / b_{\vartheta}\right|^{1 / 2}$. We may therefore take $r_{\vartheta} \sim\left|a_{\vartheta} / b_{\vartheta}\right|^{1 / 2}$ or, alternatively, $\epsilon \sim\left|b_{\vartheta} r_{d}^{2} / a_{\vartheta}\right|$. The magnitude of $b_{\vartheta}$ is governed by the remaining terms in (3.15).

Perhaps surprisingly, the magnitudes of $b_{\vartheta}$ and $r_{\vartheta}$ can be simply related to a single magnitude, that of the vertical component $B_{z}$ of the magnetic field. This is because the velocity and magnetic fields of the leading-order, semi-analytical solutions have components that are all simply related to $B_{z}$, thanks to the horizontally self-similar structure. Let $\mathrm{B}$ be the dimensional magnitude of $B_{z}$ at the bottom of the confinement layer, in units of Alfvén speed. Then the corresponding dimensionless magnitude of $B_{z}$ is $\Lambda^{1 / 2}$, where

$$
\Lambda=\frac{\mathrm{B}^{2}}{2 \Omega_{i} \eta},
$$


an Elsasser number based on $\mathrm{B}$. We assume that $\Lambda^{1 / 2}$ characterizes the order of magnitude of $B_{z}$ in the bulk of the confinement layer, and that $\partial / \partial z \sim 1$ as before. $\dagger$ Then the leading-order, semi-analytical solutions satisfy the dimensionless scaling relations

$$
\begin{aligned}
u_{z} & \sim 1, \\
u_{r} & \sim r, \\
u_{\phi} & \sim r \Lambda^{-1}, \\
B_{z} & \sim \Lambda^{1 / 2}, \\
B_{r} & \sim r \Lambda^{1 / 2}, \\
B_{\phi} & \sim r \Lambda^{-1 / 2},
\end{aligned}
$$

and these relations are expected to apply at least for $r \ll r_{\vartheta}$, and probably also for $r \lesssim r_{\vartheta}$ as a guide to orders of magnitude. They can be derived alternatively from scaling arguments applied directly to (3.14) along with the steady-state versions of (3.6)-(3.8). We note that, since the horizontal components of $\boldsymbol{B}$ increase with $r$, the total magnetic field strength $|\boldsymbol{B}|$ can greatly exceed $\mathrm{B}$ for $r \gg 1$.

For increasing values of $\Lambda$ the field lines become stiffer, so that both they and the velocity streamlines spiral less tightly, as noted in WM07. The magnitude of $\Lambda$ is not well constrained by observations, depending as it does on the magnitude of the interior field at the top of the radiative envelope. Fortunately, however, it will be found that the confinement-layer regime described here can accommodate a considerable range of $\Lambda$ values.

Now in (3.15), with the term in $\alpha_{\mu}$ neglected, the term in $\alpha_{\vartheta}$ cannot exceed the largest of the other terms in order of magnitude. From (5.3) we have $\partial \vartheta^{\prime} / \partial r \sim b_{\vartheta} r$ so that the term in $\alpha_{\vartheta}$ has magnitude $\sim \alpha_{\vartheta} b_{\vartheta} r$, at least for $r \ll r_{\vartheta}$. The remaining terms in (3.15) have magnitudes either $\sim r \Lambda^{-1}$ (the terms in $u_{\phi}$ and $B_{\phi}^{2}$ ) or $\sim r \Lambda$ (the last term). Those magnitudes follow from the horizontally self-similar structure of the semi-analytical solutions, along with $\partial / \partial z \sim 1$ and the magnitudes (5.5)-(5.10). We may therefore define the typical magnitude of $b_{\vartheta}$ to be $\alpha_{\vartheta}^{-1} \max \left(\Lambda, \Lambda^{-1}\right)$. Correspondingly, with $a_{\vartheta} \sim \eta / \kappa$ we may define $r_{\vartheta}^{2}$, the typical magnitude of $a_{\vartheta} / b_{\vartheta}$, to be

$$
r_{\vartheta}^{2}=\frac{\alpha_{\vartheta} \eta}{\kappa} \min \left(\Lambda, \Lambda^{-1}\right)=\frac{N_{\vartheta}^{2} \delta^{2}}{2 \Omega_{i} \kappa} \min \left(\Lambda, \Lambda^{-1}\right),
$$

since $\alpha_{\vartheta}=N_{\vartheta}^{2} \delta^{2} / 2 \Omega_{i} \eta$. For realistic $N_{\vartheta} \approx 0.8 \times 10^{-3} \mathrm{~s}^{-1}$, for downwelling $U \sim 10^{-5} \mathrm{~cm} \mathrm{~s}^{-1}$, and for $\Lambda \sim 1$, we have $\delta \sim 0.4 \mathrm{Mm}$ and $r_{\vartheta} \sim 4 \times 10^{3}$, corresponding to quite a large dimensional colatitudinal distance $r_{\vartheta} \delta \sim 1600 \mathrm{Mm}$.

Note incidentally that, even when the tilting is significant, such as to become incompatible with (5.1), the slopes of the thermal stratification surfaces are still geometrically small - far smaller than the geometrical aspect ratio $r_{\vartheta}^{-1}$. Indeed, even on a global scale we expect the stratification surfaces to depart from the horizontal by only 'a very tiny fraction of a megametre' from pole to equator (McIntyre 2007, end of $\S 8.5$ ), based on observational constraints on shear in the tachocline. Here of course 'horizontal' means tangential to the heliopotentials, i.e. to the sum of the centrifugal

$\dagger$ These scaling assumptions are consistent with the example solution shown in figure 3 , for which we imposed $B_{r} / r=1$ below the confinement layer, implying that $B_{z}$ is of order unity within and just below the confinement layer. Taking $z=0$ as the bottom of the confinement layer in figure 3 , we read off $B_{z}=\Lambda^{1 / 2} \approx 1.9$. Consistently, the numerical output gives $\Lambda \approx 3.5$. 
and gravitational potentials. The validity of the foregoing scale analysis, and that of the next section, will be independently checked by the numerical solutions in $\S 8$.

Of course our cylindrical model with its assumption of uniform downwelling will itself cease to apply, almost certainly, well inside such large distances from the pole. One reason for this limitation is that the cylindrical coordinates, regarded as distorted spherical coordinates, become increasingly inaccurate at large distances from the pole. However, that is not the most serious limitation. Using true spherical coordinates would not qualitatively alter the dynamics of the confinement layer. It is the assumption of uniform downwelling that places the more serious limitation on the range of applicability of our model. At some colatitude the downwelling from the real tachocline must give way to upwelling, as required by mass conservation. The confinement-layer regime cannot then apply even qualitatively. Instead, the interior magnetic field lines are free to advect and diffuse upward until they encounter the magnetic flux pumping associated with the convective overshoot layer, as assumed in figure $1(b)$. This has wider implications to be discussed in $\S 11$, including implications for lithium burning.

The range of interior field strengths accommodated by the confinement-layer regime is determined by (5.11). For uniform downwelling $U$, the condition for the regime to apply quantitatively within, say, $10^{\circ}$ colatitude or $90 \mathrm{Mm}$ of the poles is $r_{\vartheta} \delta \gg 90 \mathrm{Mm}$. We assume qualitative applicability for $r_{\vartheta} \delta \gtrsim 90 \mathrm{Mm}$. We can use (5.11) together with realistic $N_{\vartheta}$ and diffusivity values to write this last condition as

$$
\max \left(\Lambda, \Lambda^{-1}\right) \lesssim 3 \times 10^{2}\left(\frac{U}{10^{-5} \mathrm{~cm} \mathrm{~s}^{-1}}\right)^{-4}
$$

So for $U \sim 10^{-5} \mathrm{~cm} \mathrm{~s}^{-1}$ we expect the regime to apply qualitatively over a range of more than four decimal orders of magnitude in $\Lambda$. The corresponding range of field strengths, being proportional to $\Lambda^{1 / 2}$, covers more than two decimal orders of magnitude. To relate this to global-scale interior field strengths, we estimate $\left|B_{r}\right|$ from (5.9) at a nominal $30^{\circ}$ colatitude, with $\delta \approx 0.4 \mathrm{Mm}$ so that $r \approx 650$. Converting to dimensional units, we see that the range of field strengths is roughly 3 gauss $\lesssim\left|B_{r}\right| \lesssim 10^{3}$ gauss, near the top of the radiative envelope at $30^{\circ}$ colatitude. It is noteworthy that these values lie substantially above the threshold, more like $10^{-2}$ gauss, for the field strength required to enforce the Ferraro constraint in the interior over the Sun's lifetime (e.g. Mestel \& Weiss 1987; Charbonneau \& MacGregor 1993). For smaller values of $U$, a still wider range of interior field strengths becomes possible.

\section{The helium sublayer}

In this section we show that the flow through the helium sublayer has the character of flow through a porous medium. We also show that, over a large range of $N_{\mu}^{2}$ and $\alpha_{\mu}$ values, the tilting of the compositional stratification surfaces in the sublayer is even less significant than that of the thermal stratification surfaces in the confinement layer.

The helium sublayer marks the transition between the compositionally wellventilated confinement layer and the nearly impermeable, compositionally stratified helium settling layer. Therefore, we expect the dimensional sublayer thickness scale, $\delta_{\chi}$ say, to be determined by a balance between advection and diffusion of helium. In (3.10) the advection operator $\boldsymbol{u} \cdot \nabla$ scales like the strain rate $\sim U / \delta$ just above and within the sublayer, because $u_{z} \rightarrow 0$ just beneath. The strain rate $U / \delta$ must therefore 
be comparable to the helium diffusion rate $\chi / \delta_{\chi}^{2}$. Since $U / \delta=\eta / \delta^{2}$,

$$
\delta_{\chi} \sim(\chi / \eta)^{1 / 2} \delta \approx \frac{1}{7} \delta
$$

for realistic solar parameters.

Although the sublayer thickness scale $\delta_{\chi}$ is small in magnitude relative to $\delta$, it nevertheless greatly exceeds the Ekman thickness scale $\delta_{E k}=\left(v / 2 \Omega_{i}\right)^{1 / 2}$. Specifically,

$$
\frac{\delta_{\chi}^{2}}{\delta_{E k}^{2}} \sim \frac{\chi \delta^{2}}{\eta} \frac{2 \Omega_{i}}{v} \sim \frac{\chi \delta}{U} \frac{2 \Omega_{i}}{v} \sim \frac{\chi}{v} R o^{-1} \gg 1,
$$

because $\chi / \nu \approx 0.3$ while $R o^{-1} \gg 1$, typically by many decimal orders of magnitude; recall (3.12). The relations (6.1) and (6.2) suggest that the dynamics of the helium sublayer should be well described by the asymptotic regime

$$
\delta_{E k} \ll \delta_{\chi} \ll \delta \text {. }
$$

We assume (6.3) throughout this section.

Under (6.3) the magnetic diffusion rate $\eta / \delta_{\chi}^{2}$ in the sublayer greatly exceeds the helium diffusion rate $\chi / \delta_{\chi}^{2}$, by a factor $\eta / \chi \sim\left(\delta / \delta_{\chi}\right)^{2}$. The flow within the sublayer can therefore induce only a small perturbation $\boldsymbol{B}-\boldsymbol{B}_{i}=\boldsymbol{B}^{\prime}$, say, to the interior field $\boldsymbol{B}_{i}$. In figures 2 and 4 , the field lines are hardly deflected as they cross the sublayer. We may therefore analyse the sublayer as a perturbation to the state with $\boldsymbol{u}=0$ and $\boldsymbol{B}=\boldsymbol{B}_{i}$, where $\boldsymbol{B}_{i}$ has the simple dipolar structure already assumed, with components satisfying $B_{i \phi}=0, B_{i r} / r=$ constant, and $B_{i z}$ a linear function of $z$ consistent with $\nabla \cdot \boldsymbol{B}_{i}=0$.

Any such $\boldsymbol{B}_{i}$ has $\nabla \times \boldsymbol{B}_{i}=0$ and Lorentz force $\left(\nabla \times \boldsymbol{B}_{i}\right) \times \boldsymbol{B}_{i}=0$. Using this we show in Appendix A that, in the asymptotic regime given by (6.3), the steady-state induction equation becomes simply

$$
0=B_{i z} \frac{\partial}{\partial z} \boldsymbol{u}+\frac{\partial^{2}}{\partial z^{2}} \boldsymbol{B}^{\prime}
$$

in the dimensionless variables of $\S 3$. The momentum balance (3.13) becomes

$$
\boldsymbol{e}_{z} \times \boldsymbol{u}=-\nabla \tilde{p}-\alpha_{\mu} \mu \boldsymbol{e}_{z}+B_{i z} \frac{\partial}{\partial z} \boldsymbol{B}^{\prime}
$$

Here the thermal-buoyancy term $\alpha_{\vartheta} \vartheta \boldsymbol{e}_{z}$ has been absorbed into a modified pressure gradient $\nabla \tilde{p}$, which also incorporates a gradient contribution to the Lorentz force; see Appendix A, below (A 3). Because $\delta_{\chi} \ll \delta$ and because, as verified shortly, the sublayer will prove to be sufficiently flat, we may take $B_{i z}$ to be constant throughout the sublayer. It is convenient to equate the dimensional value of this constant to $B$ in the definition (5.4) of the Elsasser number $\Lambda$. Then the dimensionless magnitude of $B_{i z}$ in the sublayer is precisely $\Lambda^{1 / 2}$. We can now integrate (6.4) to give

$$
0=\Lambda^{\frac{1}{2}} \boldsymbol{u}+\frac{\partial \boldsymbol{B}^{\prime}}{\partial z}
$$

since both $\boldsymbol{B}^{\prime}$ and $\boldsymbol{u}$ vanish beneath the sublayer. Using (6.6) we write (6.5) as

$$
\boldsymbol{e}_{z} \times \boldsymbol{u}=-\nabla \tilde{p}-\alpha_{\mu} \mu \boldsymbol{e}_{z}-\Lambda \boldsymbol{u} .
$$

The term $-\Lambda \boldsymbol{u}$ has the form of a Darcy or Rayleigh drag, showing that the sublayer behaves like a porous medium on the time scale set by the strain flow. The impermeability of the helium settling layer together with the sublayer's flatness 
and thinness act to keep the flow nearly horizontal, compelling it to push past, and slightly deflect, the magnetic field lines spanning the sublayer at angles steep by comparison with sublayer aspect ratios. So the Lorentz force from the deflected field lines resists the sublayer flow in the manner of a Darcy friction; similar behaviour occurs in Hartmann layers (e.g. Debnath 1973), although in such cases viscosity also contributes to the balance of forces. Within our sublayer, by contrast, viscosity is wholly negligible provided that

$$
\Lambda \gg \delta_{E k}^{2} / \delta_{\chi}^{2}
$$

that is, provided that the Darcy friction from the field lines dominates the fluid friction from viscosity. This condition is easily satisfied, in virtue of (6.2).

In this Darcy regime, (3.14) and (3.15) simplify to

and

$$
\begin{aligned}
u_{r} & =-\Lambda u_{\phi} \\
\frac{\partial u_{\phi}}{\partial z} & =-\alpha_{\mu} \frac{\partial \mu}{\partial r}+\Lambda \frac{\partial u_{r}}{\partial z} .
\end{aligned}
$$

Together with (3.6) and (3.10), (6.9) and (6.10) describe the sublayer dynamics to an order of accuracy that includes the first corrections to perfect flatness.

We now use scaling arguments, paralleling those in $\S 5$, to verify that the corrections can indeed be taken as small and the sublayer treated as flat. As before, we expect that each term in (6.10) is proportional to $r$ and that

$$
\mu=a_{\mu}+b_{\mu} r^{2}
$$

to leading order close to the pole, where $r$ is again dimensionless and where $a_{\mu}$ and $b_{\mu}$ are dimensionless functions of $z$ alone. The matching to the helium settling layer beneath implies that $\mathrm{d} a_{\mu} / \mathrm{d} z \sim 1$ and that $a_{\mu} \sim \delta_{\chi} / \delta$ in the sublayer, if we take the constant value of $\mu$ above the sublayer to be zero. The condition for validity of flat, horizontally self-similar sublayer solutions is $r^{2} \ll r_{\mu}^{2}$, say, where $r_{\mu}$ denotes a typical magnitude of $\left|a_{\mu} / b_{\mu}\right|^{1 / 2}$. by

Within the sublayer, the pattern of dimensionless scalings (5.5)-(5.10) is replaced

$$
\begin{aligned}
u_{z} & \sim \delta_{\chi} / \delta, \\
u_{r} & \sim r, \\
u_{\phi} & \sim r \Lambda^{-1}, \\
B_{z}^{\prime} & \sim \Lambda^{1 / 2}\left(\delta_{\chi} / \delta\right)^{2}, \\
B_{r}^{\prime} & \sim r \Lambda^{1 / 2} \delta_{\chi} / \delta, \\
B_{\phi}^{\prime} & \sim r \Lambda^{-1 / 2} \delta_{\chi} / \delta,
\end{aligned}
$$

for all $r \ll r_{\mu}, r_{\vartheta}$. These dimensionless order-of-magnitude relations follow from the matching to the confinement layer, again noting its horizontally self-similar structure, and from $\nabla \cdot \boldsymbol{B}^{\prime}=\nabla \cdot \boldsymbol{u}=0$ together with (6.6) and (6.9), with $\partial / \partial z \sim \delta / \delta_{\chi} \gg 1$. Again, further detail is given in Appendix A.

The horizontal velocity components in (6.13)-(6.14) inherit their magnitudes directly from those in the overlying confinement layer. For this reason, the vertical shear in the sublayer is larger than that in the confinement layer by a factor $\delta / \delta_{\chi}$. This shows how, in the limit $\delta_{\chi} \rightarrow 0$, preserving (6.3), the sublayer regime goes over into the slip regime analysed in WM07. The slip regime has infinite shear at the top of the helium settling layer, with finite discontinuities in both horizontal velocity components. 
We can now estimate $r_{\mu}$ and hence the flatness of the sublayer, from (6.10), on the same basis as before, namely consistency with (3.10) and uniform downwelling. From (6.11) we have $\partial \mu / \partial r \sim b_{\mu} r$ for all $r \ll r_{\mu}$, so that in (6.10) the $\alpha_{\mu}$ term has magnitude $\sim \alpha_{\mu} b_{\mu} r$. Using (6.12)-(6.17) we find that the other terms in (6.10) have magnitudes $\sim r\left(\delta / \delta_{\chi}\right) \Lambda^{-1}$ (the term in $u_{\phi}$ ) and $\sim r\left(\delta / \delta_{\chi}\right) \Lambda$ (the term in $u_{r}$ ). The typical magnitude of $\alpha_{\mu} b_{\mu}$ can therefore be taken to be $\left(\delta / \delta_{\chi}\right) \max \left(\Lambda, \Lambda^{-1}\right)$. Correspondingly, since $a_{\mu} \sim \delta_{\chi} / \delta$, we may define $r_{\mu}^{2}$, the typical magnitude of $a_{\mu} / b_{\mu}$, to be $\alpha_{\mu}\left(\delta_{\chi} / \delta\right)^{2} \min \left(\Lambda, \Lambda^{-1}\right)=\alpha_{\mu}(\chi / \eta) \min \left(\Lambda, \Lambda^{-1}\right)$. Comparing this with (5.11) and recalling that $\alpha_{\mu}=N_{\mu}^{2} \delta^{2} / 2 \Omega_{i} \eta$ we see that

$$
r_{\mu}^{2}=\frac{\alpha_{\mu}}{\alpha_{\vartheta}} \frac{\kappa \chi}{\eta^{2}} r_{\vartheta}^{2}=\frac{N_{\mu}^{2}}{N_{\vartheta}^{2}} \frac{\kappa \chi}{\eta^{2}} r_{\vartheta}^{2} .
$$

Since $\kappa \chi / \eta^{2} \approx 0.75 \times 10^{3}$, we see that $r_{\mu}^{2} \gtrsim r_{\vartheta}^{2}$ for a large range of $N_{\mu}^{2}$ values, from today's value $\sim N_{\vartheta}^{2}$ down to almost three decimal orders of magnitude less. This means that, in most cases, our flatness assumptions hold even more strongly for the sublayer than for the confinement layer, in the sense of compatibility with uniform downwelling $u_{z}=u_{z}(z)$ in (3.10).

It is worth going beyond scale analysis to say more about the vertical structure of the sublayer, especially in its lower extremity or 'subtail', wherein we expect $|\boldsymbol{u}|$ to decay exponentially with depth. Within this subtail, the helium settling layer suffers only small perturbations to its otherwise uniform compositional stratification $\partial \mu / \partial z=-1$. We denote the perturbation to $\mu$ by $\mu^{\prime}$. In the steady state, (3.10) may then be approximated as

$$
-u_{z}=\frac{\chi}{\eta} \frac{\partial^{2} \mu^{\prime}}{\partial z^{2}}
$$

which can be combined with (3.6), (6.9), and (6.10) with $\mu$ replaced by $\mu^{\prime}$, to yield a single equation for $\mu^{\prime}$,

$$
\alpha_{\mu}(\eta / \chi) \nabla_{H}^{2} \mu^{\prime}=\left(\Lambda+\Lambda^{-1}\right) \frac{\partial^{4} \mu^{\prime}}{\partial z^{4}}
$$

where $\nabla_{H}^{2}=r^{-1} \partial(r \partial / \partial r) / \partial r=\nabla^{2}-\partial^{2} / \partial z^{2}$. It is now clear that the leading-order scale analysis given above applies only to the main part of the sublayer and not to the subtail. Equation (6.20) tells us that the vertical scale, $\delta_{\ell}$ say, for the subtail must depend on the horizontal scale in a manner reminiscent of the heuristic boundary-layer analysis given in GM98.

For instance if we assume that the actual horizontal scale is the scale $r_{\vartheta}$ set by the confinement layer, so that $\alpha_{\mu} \nabla_{H}^{2} \sim \alpha_{\mu} r_{\vartheta}^{-2}$, then a straightforward scale analysis of (6.20) shows that, in terms of the definitions (5.11) and (6.18),

$$
\delta_{\ell} \sim\left(\frac{r_{\vartheta}}{r_{\mu}}\right)^{\frac{1}{2}} \delta_{\chi},
$$

which is generally smaller than $\delta_{\chi}$. Even at this scale, however, viscosity remains negligible provided that the Darcy friction from the field lines dominates the fluid friction from microscopic viscosity. That is, viscosity remains negligible provided that

$$
\Lambda \gg E k \nabla^{2} \sim E k\left(\delta / \delta_{\ell}\right)^{2},
$$

equivalently

$$
\delta_{\ell}^{2} \gg \delta_{E k}^{2} / \Lambda \text {. }
$$


For realistic solar parameters, (6.22) is easily satisfied because $E k \sim 0.4 \times 10^{-8}$. A more detailed analysis (Wood 2010) verifies all these properties of the subtail. The exponentially weak flow within the subtail is invisible in figure 2, and barely visible in figure 3.

The actual horizontal scale may or may not be set by the value of $r_{\vartheta}$ since, along with boundary conditions for (6.20), it will depend on the size of the downwelling region and indeed on how the entire global picture sketched in figure $1(b)$ fits together, a point to which we return in $\S 11$.

\section{Boundary conditions for the numerical solutions}

To go beyond the self-similar, perfectly flat solutions described in $\S 4$ we must compute solutions numerically. To this end a numerical code has been written to solve the axisymmetric version of (3.5)-(3.10) in a cylindrical domain of radius $r=r_{d}$, say. The scheme on which the code is based is summarized in Appendix B. For reasons explained there and in Appendix C, it proves necessary to use the full time-dependent equations (3.5)-(3.10), rather than assuming magnetostrophic balance.

To solve the equations numerically in the cylindrical domain, we need to specify boundary conditions. This inevitably involves artificial choices. The only way to avoid making such choices would be to fit the polar caps into the complete, and highly complicated, global picture. That remains a challenge for the future, requiring the quantification of turbulent processes in the tachocline and convection zone. Such quantification would have to include realistic descriptions of turbulent magnetic flux pumping and of turbulent magnetic diffusion in the bulk of the tachocline. Also crucial is the turbulent gyroscopic pumping of polar downwelling and of the complementary upwelling in lower latitudes, together with the effects on the global-scale pattern of heat flow and the resulting feedback on $N_{\vartheta}$ distributions.

As already noted, in the present work we are imposing a dipolar magnetic field structure underneath the confinement layer, and a uniform downwelling of magnitude $U$ from a field-free region aloft. Field-free refers to time-averaged fields. In the example shown in figure 2 , the numerical domain was defined by $0 \leqslant r \leqslant 5$, i.e. $r_{d}=5$, and $-1 \leqslant z \leqslant 6$, one dimensionless unit taller than shown in the figure. We imposed $u_{z}=-1$ at $z=6$ and $B_{r} / r=1$ at $z=-1$.

As shown in $\S 4$, the bulk of the confinement layer is relatively insensitive to conditions within the field-free region aloft, and in particular to the vertical shear $\partial u_{\phi} / \partial z$ aloft. There, the vertical shear is tied to the temperature distribution via (3.16), and hence to the global-scale heat flow. To avoid having to solve the complete global-scale problem we simply imposed $\vartheta=$ const. and $\partial u_{\phi} / \partial z=0$ at $z=6$, which is consistent with the imposed uniform downwelling and also ensures that no viscous torque is exerted on the top of the domain.

At the periphery of the domain, the artificial cylindrical surface $r=r_{d}=5$, the numerical algorithm requires us to impose three vertical profiles, including the vertical profile of Maxwell stress. The stress profile represents the field lines' connection to lower latitudes and the Alfvénic torque exerted therefrom. We also need to impose thermal and compositional stratification profiles $\vartheta(z)$ and $\mu(z)$ at the periphery, in a manner consistent with scalings in the confinement layer and helium sublayer ( $\S 5$ and 6). In this way we artificially fix the altitude of the helium sublayer. We thereby influence the velocity field as well, since it is tightly linked to the two stratifications by (3.9) and (3.10). The remaining peripheral boundary conditions are used to promote smoothness of the fields, and hence to minimize spurious boundary effects in the 
steady state. In particular, we impose

and

$$
\begin{aligned}
\frac{\partial}{\partial r}\left(\frac{u_{r}}{r}\right) & =0, \\
\frac{\partial}{\partial r}\left(\frac{u_{\phi}}{r}\right) & =0, \\
\frac{\partial B_{z}}{\partial r} & =0
\end{aligned}
$$

at $r=r_{d}=5$.

At the bottom of the domain, the horizontal surface $z=-1$, we impose the conditions (3.1) and (3.2), equivalently $\partial \vartheta / \partial z=1$ and $\partial \mu / \partial z=-1$. We also impose $u_{\phi}=0$, i.e. that the interior is in solid rotation, with dimensional angular velocity $\Omega_{i}$, as required by the global picture. As discussed at the end of $\S 3$ the global picture also requires that $B_{\phi}=0$, i.e. that there is no Alfvénic torque, on the bottom of the domain. This last condition cannot be directly imposed, however. Instead, it must be approached via iterative adjustments to the Maxwell stress profile at the periphery $r=r_{d}$. The iteration procedure and its rationale are described in $\S 8$.

We can now see more clearly why $u_{z}(z)$ could be specified arbitrarily when constructing the semi-analytical solutions in $\S 4$. As already mentioned, for the numerical solution we need to specify three vertical profiles at the periphery $r=r_{d}$, which are taken to be $\vartheta(z), \mu(z)$ and $B_{\phi}(z)$. For the semi-analytical solutions of $\S 4$, $\vartheta(z)$ and $\mu(z)$ could not be specified independently. Rather, they were both determined by (4.1), up to boundary conditions, as soon as $u_{z}(z)$ was specified. We could still have specified $B_{\phi}(z)$, but gave up that freedom in order to ensure the vanishing of the Alfvénic torque as $z \rightarrow-\infty$, thus determining $B_{\phi}(z)$ via the expression (4.9). Also allowed by the semi-analytical framework was the freedom to specify $u_{\phi}(z)$ at $r=r_{d}$, which we similarly gave up in order to ensure solid rotation as $z \rightarrow-\infty$, thus determining $u_{\phi}(z)$ via (4.11).

More generally, within the semi-analytical framework, the peripheral and bottom profiles of $B_{\phi}$ contain equivalent information, and similarly for $u_{\phi}$. This is because of the Alfvénic coupling along the field lines linking the periphery to the bottom of the domain, expressed by the $\boldsymbol{B} \cdot \nabla$ operator in (4.2) and (4.4). There is no such precise equivalence within the numerical framework. The time-dependence, in the equations solved numerically, replaces static Alfvénic coupling by Alfvénic wave propagation, requiring one peripheral and one bottom profile to be specified, which we take to be $B_{\phi}(z)$ and $u_{\phi}(r)$ respectively. This is analogous to the need for boundary conditions at both ends of a stretched string in motion.

\section{The numerical solutions}

Computing limitations preclude a perfect match to the real Sun's parameter values. They also require a slight modification to (3.5)-(3.10), explained in Appendix B, in which artificial horizontal diffusivities $v_{H}, \chi_{H}$ are introduced. These maintain numerical stability while allowing small enough $v$ and $\chi$ in the important vertical diffusion terms.

From the scale analyses in $\S \S 5$ and 6 we may identify the conditions most essential to reaching a qualitatively similar parameter regime - that is, qualitatively similar to a regime with a perfect parameter match to the real Sun. Those essential conditions are: 


$\begin{array}{lccc}\begin{array}{l}\text { Dimensionless } \\ \text { parameter }\end{array} & \begin{array}{c}\text { Nominal } \\ \text { solar value }\end{array} & \begin{array}{c}\text { Value for } \\ \text { numerical solution }\end{array} & \text { Condition } \\ R o & 5 \times 10^{-8} & 10^{-2} & \ll 1 \\ \kappa / \eta & 3 \times 10^{4} & 10^{2} & \gg 1 \\ \alpha_{\vartheta}(\eta / \kappa) & 1.4 \times 10^{7} & 50 & >\max \left(\Lambda, \Lambda^{-1}\right)^{\dagger} \\ \left(r_{\mu} / r_{\vartheta}\right)^{2} & 3 \times 10^{2} & 2 & >1 \\ \chi / \eta & 2 \times 10^{-2} & 2 \times 10^{-2} & \ll 1 \\ \nu / \chi & 3 & 1 & \ll 1 / R o \\ \text { † This condition corresponds to } r_{\vartheta}^{2}>1, \text { with } r_{\vartheta}^{2}=\alpha_{\vartheta}(\eta / \kappa) \min \left(\Lambda, \Lambda^{-1}\right) \text { as defined in }(5.11) .\end{array}$

TABLE 1. Parameter values and conditions; see text.

(i) The Rossby number Ro should be small in comparison with unity, so that the steady state is close to magnetostrophic.

(ii) The thermal diffusivity $\kappa$ should be large in comparison with the magnetic diffusivity $\eta$, so that the confinement-layer flow only weakly perturbs the background thermal stratification.

(iii) The confinement layer and helium sublayer should both be reasonably flat, to the extent that $r_{\vartheta}$ and $r_{\mu}$ are both distinctly greater than 1. With (6.18) in mind, we also take $r_{\mu}>r_{\vartheta}$.

(iv) The helium diffusivity $\chi$ should be small in comparison with the magnetic diffusivity $\eta$, so that the helium sublayer is thinner than, and therefore distinct from, the magnetic confinement layer.

(v) The viscosity $v$ should be small enough that an Ekman layer does not form at the top of the helium settling layer, so that the flow is everywhere inviscid, even in the helium sublayer. With small Ro this condition is easily satisfied in the numerical model as well as in the real Sun, because of the factor $R o^{-1}$ in (6.2).

Leaving $v_{H}$ and $\chi_{H}$ aside for the moment (see Appendix B) we can characterize the system by seven dimensionless parameters, including the Elsasser number $\Lambda$, which enters through the boundary conditions. Table 1 presents the other six dimensionless parameters, with nominal solar values alongside the values used for the numerical solution presented here (figures 2, 5, and 6). The last column echoes aspects of the qualitative parameter conditions just stated. The nominal solar values assume $U=10^{-5} \mathrm{~cm} \mathrm{~s}^{-1}$.

In order to allow the stratification surfaces to develop a significant tilt, the horizontal size of the numerical domain, $r_{d}$, was chosen to be of the same order as $r_{\vartheta}$; specifically, we chose $r_{d}=5$. However, the precise value of $r_{\vartheta}$, as determined by (5.11), depends on the precise value of $\Lambda$, which is set indirectly via the boundary condition for $B_{r}$. For the case shown in figures 2,5 , and 6 this boundary condition was $B_{r} / r=1$ at $z=-1$, and we find from the solution that $\Lambda=\left.B_{i z}^{2}\right|_{z=0} \approx 3.5$. With the parameter values in the second-last column of table 1 , this in turn means that $r_{\vartheta}^{2} \approx 50 / 3.5 \approx 14$, so in fact $r_{\vartheta}$ is slightly smaller than $r_{d}$. Nevertheless the numerical solution is remarkably close to being flat, even close to the periphery of the domain $r=r_{d}$. Some effects of departures from flatness can be seen near the periphery in, for instance, figure 10 below, but they do not qualitatively alter the nature of the flow. The confinementlayer dynamics can therefore apply, at least qualitatively, even at colatitudes for which the semi-analytical solutions of $\S 4$ are not strictly valid. We should therefore regard (5.12) as a conservative estimate of the range of interior field strengths for which the confinement-layer regime applies. 
(a)

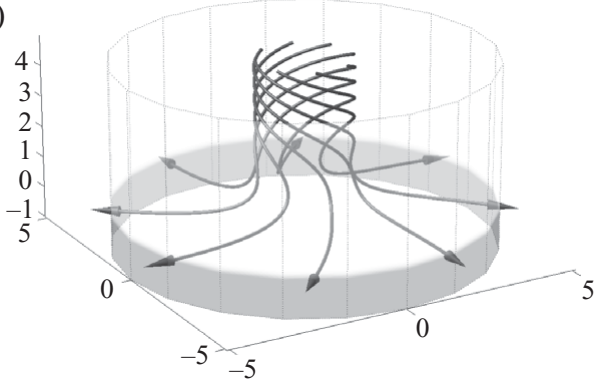

(b)

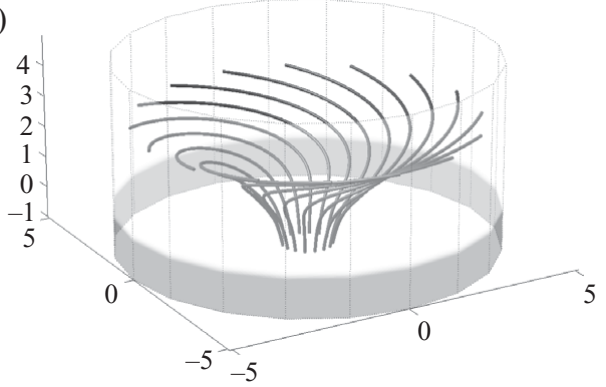

FIGURE 5. A numerical solution of the confinement-layer equations with $r_{\vartheta}^{2} \approx 14$ and Elsasser number $\Lambda \approx 3.5$. Other parameter values are given in the second-last column of table 1 . From the same solution as shown in figure 2 . Both here and in figure 2 , a top layer $5 \leqslant z \leqslant 6$ has been omitted from the plots. Figures 2, 5, and 6 are all views of the same numerical solution.
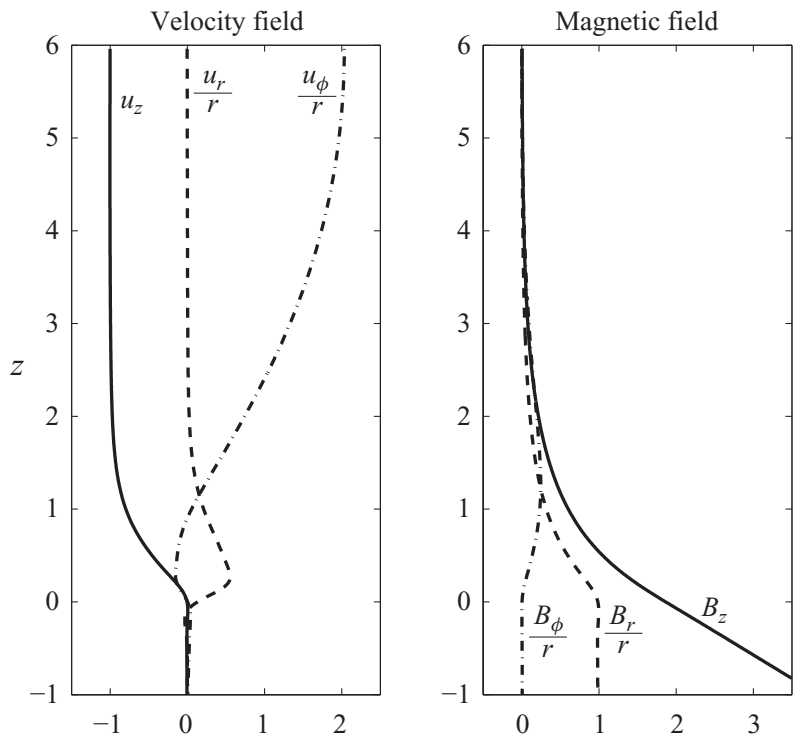

FIGURE 6. Vertical profiles from a numerical solution of the confinement-layer equations with $r_{\vartheta}^{2} \approx 14$ and Elsasser number $\Lambda \approx 3.5$. Other parameter values are given in the second-last column of table 1 . From the same solution as shown in figure 2 .

The vertical profiles of $\vartheta, \mu$ and $B_{\phi}$ at the periphery of the computational domain were initially taken from a semi-analytical solution. The resulting steadystate meridional flow, and the distribution of Coriolis torque it exerts on each field line, cannot be precisely known in advance. So the steady state found, with this choice of the peripheral $B_{\phi}$ profile, will generally be such that some of the balancing Alfvénic torque is exerted on the bottom of the computational domain, rather than on the periphery $r=r_{d}$.

As mentioned in the previous section, we can eliminate this bottom torque through iterative adjustments to the torque at the periphery. Experience with the numerical solutions reveals that such adjustments are propagated along the magnetic field lines without, in most cases of interest, greatly perturbing the poloidal velocity and magnetic field components. So we can reduce the bottom torque simply by mapping or transferring that torque from the bottom to the periphery of the domain, along 
the field lines. The bottom torque is thereby reduced, though not all the way to zero since the other fields adjust, slightly reshaping the field lines. The process can then be repeated, further reducing the bottom torque. After a sufficient number of iterations, the bottom torque can be reduced to negligible values, with practically all the torque transferred to the periphery.

Figure 5 shows plots of the steady-state streamlines and magnetic field lines from the numerical solution whose parameter values are listed in table 1, and whose meridional cross-section was presented in figure 2 . The bottom torque is negligibly small. Figure 6 shows vertical profiles on the rotation axis, from the same numerical solution.

\section{Upper-domain 'slipperiness'}

On the rotation axis, where the profiles in figure 6 were taken, the stratification surfaces are flat for any finite $\alpha_{\vartheta}$ and $\alpha_{\mu}$. If the numerical solution were in perfect magnetostrophic balance then we could use its $u_{z}(z)$ profile to calculate the other field components on the axis by the procedure for constructing semi-analytical solutions described in $\S 4$. But the numerical solutions are not in perfect balance, especially toward the upper part of the domain, where the Lorentz and Coriolis forces become small and the artificial viscous forces relatively more significant, along with numerical truncation errors and other small effects. In particular, the numerical $u_{z}(z)$ and $u_{r}(z)$ profiles will not conform precisely to the decay law (4.10) as $z$ increases. So the semi-analytical solution obtained by this process cannot perfectly match the numerical solution, even on the rotation axis. Indeed, such a semi-analytical solution will often exhibit wild deviations from the numerical solution toward the upper part of the domain. There, the delicate balance of terms gives the dynamics a certain 'slipperiness', as already evidenced by the upper-domain sensitivity of $u_{\phi}$ and $\partial u_{\phi} / \partial z$ to values of the decay constant $\gamma$ in (4.10).

To enable a meaningful comparison between the numerical and semi-analytical solutions, we are therefore compelled to make small adjustments to $u_{z}(z)$ in the upper domain, to make $u_{z}(z)$ and $u_{r}(z)$ conform to (4.10), before using them to compute a semi-analytical solution. In the case shown here the decay constant $\gamma$ was chosen, purely for illustration, to be 2.24 . The required adjustment to $u_{z}(z)$ is then very small indeed; the solid $u_{z}$ curves on the left of figures 3 and 6 are practically indistinguishable from each other.

As already mentioned, conditions aloft in the real Sun may well involve small-scale MHD turbulence. The resulting departures from magnetostrophic balance may well produce asymptotic behaviour aloft that disagrees with all the solutions obtained here, whether semi-analytical or numerical. A realistic matching to conditions aloft remains a challenge for future modelling work.

\section{Confinement layers with no helium settling layer}

Although the presence of the helium settling layer influences the structure of the confinement layer in today's Sun, it is not actually essential to magnetic confinement. The qualitative picture sketched in figure $1(b)$ might therefore apply also to the early Sun, before the helium settling layer developed.

The analysis presented in $\S 4$ holds good for any suitable profile of $u_{z}(z)$, provided only that the thermal stratification is sufficiently strong. Compositional stratification enters only indirectly, via the shape of $u_{z}(z)$ in its lower part representing the helium sublayer and subtail. As pointed out below (6.21)-(6.23), the shape depends in turn 

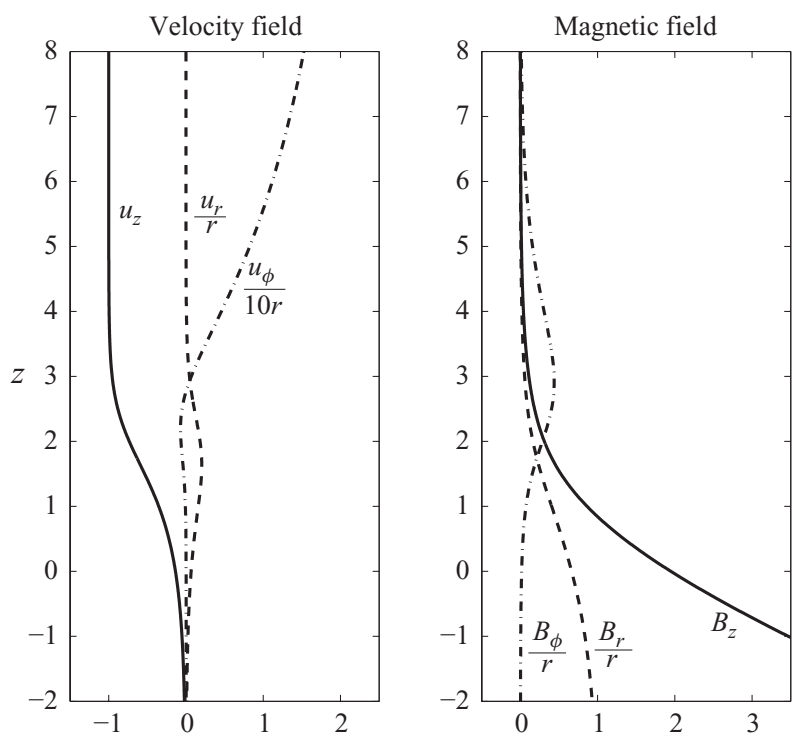

FiguRE 7. A semi-analytical solution of the confinement-layer equations with no helium settling layer. The downwelling was chosen such that $\gamma=2.24$. The $u_{\phi}$ profile has been rescaled by a factor of 10 . The $z$-origin is arbitrary.

on how the confinement layer and sublayer fit into the global picture sketched in figure $1(b)$.

For the early Sun, with no compositional stratification, we have a similar indeterminacy in the shape of $u_{z}(z)$. However, we may note that with no helium settling layer the scaling analysis in $\S 5$ then applies not only within the confinement layer, but also in the region immediately beneath. An argument similar to that leading to the estimate $\delta_{\ell}$ of the subtail scale in (6.21) predicts that $|\boldsymbol{u}|$ decays with depth on a length scale $\sim \delta$, rather than $\delta_{\ell}$.

Analytical solutions with no helium settling layer can be calculated in the same way as in $\S 4$. Figure 7 presents such a solution. In this case, the downwelling profile was chosen to be $u_{z}(z)=-(1+\exp (-\gamma(z-2)))^{-1 / \gamma}$, so that $u_{z}=O(\exp (z))$ as $z \rightarrow-\infty$ and $u_{z}=-1+O(\exp (-\gamma z))$ as $z \rightarrow+\infty$. We have taken $\gamma=2.24$, again purely for illustration, to allow a more direct comparison with figures 3 and 6 . Figure 8 shows a vertical cross-section through the solution presented in figure 7 .

\section{Conclusions and future directions}

We cannot yet claim to have a complete tachocline theory. Indeed, the confinement layer and helium sublayer form only two pieces of a complicated jigsaw puzzle. Other aspects of that jigsaw include the way in which the confinement layer matches upward to the relatively large negative shear in the bulk of the tachocline, and the way in which the baroclinic temperature anomalies induced by the tachocline's MMC fit into the perturbed global-scale heat flow. In particular, without putting the whole jigsaw together we cannot quantitatively predict the thickness of the tachocline. Nor can we predict the precise shapes of the vertical profiles of $\boldsymbol{B}$ and $\boldsymbol{u}$ in the confinement layer. Those profile shapes depend on matching to conditions not only aloft but also equatorward, where stratification surfaces and field lines extend into colatitudes outside the polar downwelling regions. 


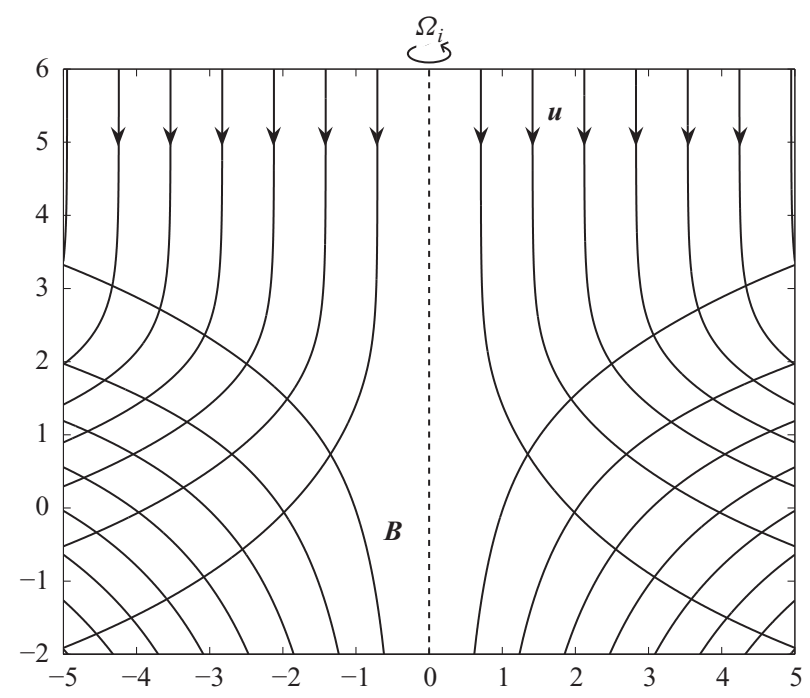

FIGURE 8. The magnetic confinement layer near the north pole in a model for the early Sun, with no helium settling layer. The plot is from the same semi-analytical solution as that of figure 7.

However, the results obtained here give us the first fully consistent model of polar field confinement, as such, together with insight into how it could work in today's Sun. The results cover a wide range of possible downwelling values and interior field strengths (end of $\S 5$; as remarked in $\S 8,(5.12)$ appears to be a conservative estimate). We have also shown, in $\S 10$, how confinement could have worked in the early Sun. The dynamics is similar apart from the slightly deeper penetration of the MMC in the absence of the helium settling layer and helium sublayer. We can use the resulting insights, alongside our well-established understanding of the gyroscopic pumping of MMCs, to say something new about the early Sun and the solar lithium-burning problem.

Standard solar-evolution models predict surface lithium abundances higher than observed by a factor $\sim 10^{2}$ (e.g. Vauclair et al. 1978). The reason is that the standard models mix material down to the bottom of the convection zone but no further. To destroy lithium, material from the convection zone must be mixed or circulated to somewhat greater depths and therefore to somewhat higher temperatures, beyond those at the bottom of today's tachocline. However, there is no evidence of depletion of the convection zone's beryllium, which is destroyed at only modestly higher temperatures than lithium. Further discussion and references may be found in Christensen-Dalsgaard, Gough \& Thompson (1992), in Wood (2010, chap. 6), and in Mestel (2011, §8.8.5). Here we argue that a quantitative version of the scenario sketched in figure $1(b)$ has promise as a way of circulating material to the required depth in the early Sun, and no further, thus making sense of the high beryllium as well as the low lithium abundance.

As already mentioned in $\S 1$, the downwelling MMC in the polar tachocline that makes field confinement possible can be regarded as due to a gyroscopically-pumped MMC trying to burrow downward, but held in check by its encounter with the interior field $\boldsymbol{B}_{i}$ and with the helium settling layer, if present. If, in a thought experiment, we were to switch off the interior field $\boldsymbol{B}_{i}$, then the downwelling would spread or burrow to ever-increasing depths. The time scale for such burrowing is inversely proportional to $\Omega_{i}^{2}$ (e.g. McIntyre 2007, (8.15)ff.); one may think of rotational stiffness as strengthening the burrowing tendency. 


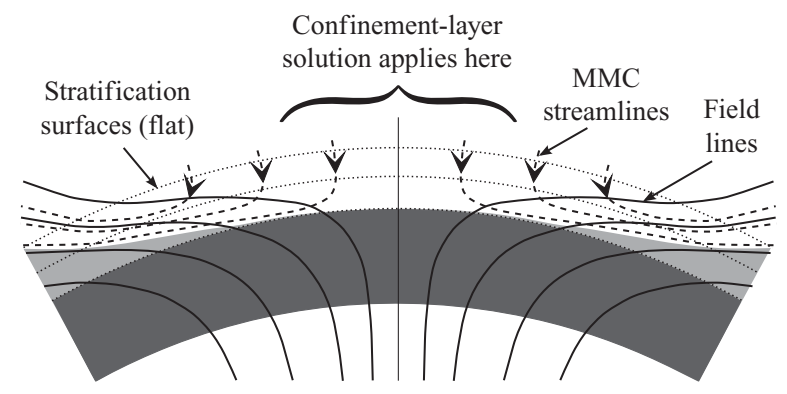

FIGURE 9. Schematic drawing of the magnetic confinement layer and its immediate surroundings at the bottom of the high-latitude tachocline. Close to the pole the interior magnetic field (solid lines) is confined by the downwelling MMC (dashed streamlines). The vertical scale has been greatly exaggerated.

Now, because the early Sun rotated much faster than today, not only would there have been no helium settling layer but, also, the burrowing tendency would have been much stronger than today, tending to push the bottom of the tachocline downward. This reopens the possibility conjectured in GM98 that there might have been a ventilated 'polar pit' in which most of the convection zone's lithium, though not too much of its beryllium, was burnt during the first gigayear or two of the Sun's main-sequence evolution.

To take this further we again need to consider the way in which the confinement layer fits into the global picture. It is arguable that the bottom of the entire polar downwelling region is depressed relative to its surroundings, forming not so much a 'pit' as a shallow 'frying pan', too shallow to burn lithium in today's Sun but possibly just deep enough in the early Sun.

Here we need to distinguish the shape of the ventilated region from the shapes of the stratification surfaces, which latter must remain relatively flat, meaning close to the horizontal. Figure 9 sketches the way in which the confinement layer might fit into its surroundings near the bottom of the polar tachocline. The stratification surfaces are shown dotted. At the periphery of the polar downwelling region, the field lines (solid) spiral outward and upward from the confinement layer on their way to lower latitudes. They will tend to splay out, as well as slanting upward, as they emerge from the downwelling region. The MMC will similarly slant upward, flowing approximately along the field lines (dashed streamlines). This is because the splaying-out increases the magnetic Reynolds number beyond the order-unity values characteristic of the confinement layer. Further out, the field lines must continue to rise through the tachocline until they encounter the convection zone's overshoot layer, where they are held horizontal by turbulent magnetic flux pumping as suggested in figure $1(b)$. On the way we must expect turbulent eddy fluxes to become increasingly important, decoupling the MMC's upwelling streamlines from the time-averaged field lines and leaving the upwelling free to spread over a wide range of latitudes, constrained only by mass conservation and global-scale heat flow.

Such a picture applies equally well to today's Sun and to the early Sun, the main difference being that the ventilated polar region (unshaded in figure 9) is likely to have been pushed deeper in the early Sun with its much faster rotation, stronger burrowing tendency, and global-scale $\left|\boldsymbol{B}_{i}\right|$ values only modestly larger. The ventilated polar regions could well have been deeper by many tens of megametres, as required to burn lithium. This deepening is additional to that of the convection zone itself, in the early Sun relative to today's Sun, amounting to several more tens of megametres according to standard solar models (e.g. Ciacio et al. 1997). The early Sun would have 
started to form its helium settling layer just below the ventilated, lithium-destroying polar regions, i.e. just below the polar confinement layers. Then, thanks to the Sun's evolution and to the gradual diminution of $\Omega_{i}$ through solar-wind braking, the ventilated regions would have retreated upward, and the top of the helium settling layer would have followed them upward as new helium strata formed.

Within the peripheral lightly-shaded region in figure 9, into which the MMC does not penetrate, we suggest that ventilation is weak or nonexistent and that shear will be limited by the Ferraro constraint. The darker shading represents the top part of today's helium settling layer.

As the lightly-shaded region expands upward and outward beyond the immediate surroundings sketched in figure 9, through the tachocline toward the overshoot layer, we may surmise that small-scale MHD instabilities kick in (e.g. Spruit 2002; Gilman \& Cally 2007; Parfrey \& Menou 2007, and references), breaking the Ferraro constraint and blurring the distinction between the shaded and unshaded regions as turbulent eddy fluxes increase. So a larger-scale picture of the 'lithium frying pan' would show its upward-sloping lower boundary becoming increasingly porous and indistinct at greater colatitudes.

The global tachocline model that would be needed to test, and to begin to quantify, the foregoing speculations would have to describe

(i) the precise way in which turbulent stresses in the convection zone and tachocline gyroscopically pump the polar downwelling needed to confine $\boldsymbol{B}_{i}$ in polar latitudes;

(ii) the global-scale distribution of temperature and heat flow that fits in with the MMCs;

(iii) the turbulent magnetic flux pumping by convective overshoot assumed to confine $\boldsymbol{B}_{i}$ in extra-polar latitudes;

(iv) the extent to which the winding-up of the time-averaged toroidal field in extra-polar latitudes of the tachocline (figure $1 b$ ) is limited by turbulent eddy fluxes;

(v) the reaction of the overlying turbulent layers to all of the above, especially the deficit in the convection zone's differential rotation governing the torques exerted from above, for instance via feedback on the strength of gyroscopic pumping of the MMC.

Progress on these formidable problems will depend on finding suitable ways to model the turbulent processes.

We thank N. H. Brummell, J. Christensen-Dalsgaard, W. Däppen, S. Degl'Innocenti, P. Garaud, D. O. Gough, D. Jault, C. A. Jones, D. Lecoanet, L. Mestel, M. Miesch, M. R. E. Proctor, T. M. Rogers, S. N. Shore, M. J. Thompson, S. M. Tobias, N. O. Weiss, L. A. Willson, and several referees for helpful and stimulating comments and discussions. T.S.W. was supported by a UK Science and Technology Facilities Council Research Studentship, and M.E.M.'s early thinking on the tachocline problem was supported by a SERC/EPSRC Senior Research Fellowship.

\section{Appendix A. Helium sublayer scalings}

For perturbations $\boldsymbol{B}^{\prime}$ to a background magnetic field $\boldsymbol{B}_{i}$, the Lorentz force may be written as

$$
\boldsymbol{F}=\left(\nabla \times\left(\boldsymbol{B}_{i}+\boldsymbol{B}^{\prime}\right)\right) \times\left(\boldsymbol{B}_{i}+\boldsymbol{B}^{\prime}\right)=-\nabla\left(\frac{1}{2}\left|\boldsymbol{B}_{i}\right|^{2}+\boldsymbol{B}_{i} \cdot \boldsymbol{B}^{\prime}+\frac{1}{2}\left|\boldsymbol{B}^{\prime}\right|^{2}\right)+\left(\boldsymbol{B}_{i}+\boldsymbol{B}^{\prime}\right) \cdot \nabla\left(\boldsymbol{B}_{i}+\boldsymbol{B}^{\prime}\right) .
$$


If, as here, the background field $\boldsymbol{B}_{i}$ is curl-free, then all the terms quadratic in $\boldsymbol{B}_{i}$ vanish and we have

$$
\boldsymbol{F}=-\nabla\left(\boldsymbol{B}_{i} \cdot \boldsymbol{B}^{\prime}+\frac{1}{2}\left|\boldsymbol{B}^{\prime}\right|^{2}\right)+\boldsymbol{B}^{\prime} \cdot \nabla \boldsymbol{B}_{i}+\left(\boldsymbol{B}_{i}+\boldsymbol{B}^{\prime}\right) \cdot \nabla \boldsymbol{B}^{\prime} .
$$

If $\boldsymbol{B}_{i}$ and $\boldsymbol{B}^{\prime}$ are axisymmetric, and have the scalings given by (6.15)-(6.17), with $\partial / \partial z \sim \delta / \delta_{\chi} \gg 1$, then all components of $\boldsymbol{B}^{\prime} \cdot \nabla \boldsymbol{B}^{\prime}$ are smaller than the corresponding components of $\boldsymbol{B}_{i} \cdot \nabla \boldsymbol{B}^{\prime}$ by factors $\left(\delta_{\chi} / \delta\right)^{2}$, with one exception. The $r$ component of $\boldsymbol{B}^{\prime} \cdot \nabla \boldsymbol{B}^{\prime}$ includes a term $B_{\phi}^{\prime 2} / r$, the divergence of the hoop stress. Relative to the $r$ component of $\boldsymbol{B}_{i} \cdot \nabla \boldsymbol{B}^{\prime}$ this term is of order $\left(\delta_{\chi} / \delta\right)^{2} / \Lambda^{2}$. However, the hoop-stress term itself is smaller than the $r$ component of the Coriolis force by a factor $\left(\delta_{\chi} / \delta\right)^{2}$, even for small $\Lambda$, because of (6.14). Therefore, in the asymptotic regime (6.3), (3.13) becomes

$$
\boldsymbol{e}_{z} \times \boldsymbol{u}=-\nabla p^{\prime}+\alpha_{\vartheta} \vartheta \boldsymbol{e}_{z}-\alpha_{\mu} \mu \boldsymbol{e}_{z}-\nabla\left(\boldsymbol{B}_{i} \cdot \boldsymbol{B}^{\prime}+\frac{1}{2}|\boldsymbol{B}|^{2}\right)+\boldsymbol{B}^{\prime} \cdot \nabla \boldsymbol{B}_{i}+\boldsymbol{B}_{i} \cdot \nabla \boldsymbol{B}^{\prime}
$$

The flow through the sublayer produces only a small perturbation to the otherwise uniform thermal stratification. From (3.9) and (3.10) we see that, within the sublayer, variations in $\vartheta$ are smaller than variations in $\mu$ by a factor $\chi / \kappa \ll 1$. The thermal stratification in the sublayer may therefore be treated as horizontally uniform, allowing the thermal buoyancy term $\alpha_{\vartheta} \vartheta \boldsymbol{e}_{z}$ in (A 3) to be incorporated into the pressure field along with the $-\nabla\left(\boldsymbol{B}_{i} \cdot \boldsymbol{B}^{\prime} \ldots\right)$ term. We denote the modified pressure field as $\tilde{p}$.

The perturbed steady-state induction equation is

$$
0=\left(\boldsymbol{B}_{i}+\boldsymbol{B}^{\prime}\right) \cdot \nabla \boldsymbol{u}-\boldsymbol{u} \cdot \nabla\left(\boldsymbol{B}_{i}+\boldsymbol{B}^{\prime}\right)+\nabla^{2} \boldsymbol{B}^{\prime},
$$

again on the assumption that $\boldsymbol{B}_{i}$ is curl-free. It is readily verified from the scalings (6.12)-(6.17) and $\partial / \partial z \sim \delta / \delta_{\chi} \gg 1$ that each component of $\boldsymbol{u} \cdot \nabla \boldsymbol{B}^{\prime}$ is of the same order as the corresponding component of $\boldsymbol{B}^{\prime} \cdot \nabla \boldsymbol{u}$, but smaller than $\boldsymbol{B}_{i} \cdot \nabla \boldsymbol{u}$ by a factor $\left(\delta_{\chi} / \delta\right)^{2}$. Furthermore, provided that the horizontal scales $r_{\vartheta}$ and $r_{\mu}$ are both $\gg 1$ we may also make the boundary-layer approximation, $\nabla^{2} \approx \partial^{2} / \partial z^{2}$. Thus, in the asymptotic regime (6.3), we may simplify (A 4) to

$$
0=\boldsymbol{B}_{i} \cdot \nabla \boldsymbol{u}-\boldsymbol{u} \cdot \nabla \boldsymbol{B}_{i}+\frac{\partial^{2}}{\partial z^{2}} \boldsymbol{B}^{\prime}
$$

If the field $\boldsymbol{B}_{i}$ were uniform, and directed along the axis of rotation, then (A 3) and (A 5) would reduce immediately to (6.5) and (6.4) respectively. Since $\boldsymbol{B}_{i}$ is axisymmetric and smooth, this reduction still holds as a first approximation within some neighbourhood of the axis. It is sufficient to show that this neighbourhood includes the entire sublayer within a radius $r \sim r_{\mu}$. We first note that, since $\nabla \cdot \boldsymbol{B}_{i}=0$, we have $B_{i r} / r=-\frac{1}{2} \partial B_{i z} / \partial z$ as in (4.8). In the sublayer we have $\partial B_{i z} / \partial z \sim B_{i z}$ (dimensionally, $\partial B_{i z} / \partial z \sim B_{i z} / \delta$ ) as a consequence of the matching to the confinement layer, as can be seen, for instance, from the solid curve in the right-hand panel of figure 3. Now applying the scalings (6.12)-(6.17) we find that, in (A 3), each component of $\boldsymbol{B}^{\prime} \cdot \nabla \boldsymbol{B}_{i}$ is smaller than the corresponding component of $\boldsymbol{B}_{i} \cdot \nabla \boldsymbol{B}^{\prime}$ by a factor $\delta_{\chi} / \delta$, and that, in (A 5), each component of $\boldsymbol{u} \cdot \nabla \boldsymbol{B}_{i}$ is smaller than the corresponding component of $\boldsymbol{B}_{i} \cdot \nabla \boldsymbol{u}$ by the same factor $\delta_{\chi} / \delta$. Moreover, even at colatitudes $r \sim r_{\mu}$ the contributions $B_{i z} \partial \boldsymbol{B}^{\prime} / \partial z$ and $B_{i z} \partial \boldsymbol{u} / \partial z$ dominate all other contributions to $\boldsymbol{B}_{i} \cdot \nabla \boldsymbol{B}^{\prime}$ and $\boldsymbol{B}_{i} \cdot \nabla \boldsymbol{u}$ by factors of at least $\delta / \delta_{\chi}$. So (A 3) and (A 5) do indeed reduce to (6.5) and (6.4).

For $r \lesssim r_{\mu}$, tilting of the compositional isopleths produces variations in the dimensionless altitude of the sublayer no greater than $O\left(\delta_{\chi} / \delta\right)$, so that $B_{i z}$ may be assumed constant within the sublayer as assumed in the derivation of (6.9) and 
(6.10). Finally, we note that the foregoing picture applies not only to steady states but also to time-dependent states with any time scale, such as $\delta / U=\delta^{2} / \eta$, that is long in comparison with the time scale $\delta_{\chi}^{2} / \eta$ for magnetic diffusion across the sublayer. On any such time scale the sublayer therefore behaves like a porous medium.

\section{Appendix B. The numerical scheme}

We wish to solve a suitable version of (3.5)-(3.10) in axisymmetric cylindrical polar coordinates. We introduce streamfunctions $\Psi$ and $A$, i.e. azimuthal vector-potential components, for the poloidal velocity and magnetic fields, such that

$$
\begin{aligned}
& u_{z}=\frac{1}{r} \frac{\partial(r \Psi)}{\partial r} \quad \text { and } \quad u_{r}=-\frac{\partial \Psi}{\partial z} \\
& B_{z}=\frac{1}{r} \frac{\partial(r A)}{\partial r} \quad \text { and } \quad B_{r}=-\frac{\partial A}{\partial z}
\end{aligned}
$$

guaranteeing that the fields are divergence-free; $r \Psi$ is sometimes called the Stokes streamfunction. The azimuthal vorticity $\omega_{\phi}$ and electric current density $J_{\phi}=(\nabla \times \boldsymbol{B})_{\phi}$ are related to $\Psi$ and $A$ by

and

$$
\begin{aligned}
\omega_{\phi} & =-\left(\nabla^{2}-r^{-2}\right) \Psi \\
J_{\phi} & =-\left(\nabla^{2}-r^{-2}\right) A .
\end{aligned}
$$

As already explained, and further discussed below, we introduce anisotropic viscosity and helium diffusivity with dimensionless horizontal components $v_{H}$ and $\chi_{H}$. So (3.5)-(3.10) are replaced by

$$
\begin{aligned}
R o \frac{1}{r} \frac{\mathrm{D}\left(r u_{\phi}\right)}{\mathrm{D} t}-\frac{\partial \Psi}{\partial z} & =\frac{1}{r} \boldsymbol{B} \cdot \nabla\left(r B_{\phi}\right)+E k\left[\frac{\partial^{2}}{\partial z^{2}}+v_{H}\left(\nabla_{H}^{2}-\frac{1}{r^{2}}\right)\right] u_{\phi}, \\
R o\left[r \frac{\mathrm{D}\left(\omega_{\phi} / r\right)}{\mathrm{D} t}+\frac{\partial\left(r u_{\phi}, u_{\phi} / r\right)}{\partial(z, r)}\right]-\frac{\partial u_{\phi}}{\partial z}=-\alpha_{\vartheta} \frac{\partial \vartheta}{\partial r}+\alpha_{\mu} \frac{\partial \mu}{\partial r}+r \boldsymbol{B} \cdot \nabla\left(J_{\phi} / r\right) & \quad+\frac{\partial\left(r B_{\phi}, B_{\phi} / r\right)}{\partial(z, r)}+E k\left[\frac{\partial^{2}}{\partial z^{2}}+v_{H}\left(\nabla_{H}^{2}-\frac{1}{r^{2}}\right)\right] \omega_{\phi}, \\
r \frac{\mathrm{D}\left(B_{\phi} / r\right)}{\mathrm{D} t} & =r \boldsymbol{B} \cdot \nabla\left(u_{\phi} / r\right)+\left(\nabla^{2}-\frac{1}{r^{2}}\right) B_{\phi}, \\
\frac{1 \mathrm{D}(r A)}{r} & =\left(\nabla^{2}-\frac{1}{\mathrm{D}^{2}}\right) A, \\
\frac{\mathrm{D} \vartheta}{\mathrm{D} t} & =\frac{\kappa}{\eta} \nabla^{2} \vartheta \\
\frac{\mathrm{D} \mu}{\mathrm{D} t} & =\frac{\chi}{\eta}\left[\frac{\partial^{2}}{\partial z^{2}}+\chi_{H} \nabla_{H}^{2}\right] \mu .
\end{aligned}
$$

We solve these equations using a simple finite-difference scheme on an Eulerian grid regularly spaced in $r$ and $z$ at intervals $\Delta r$ and $\Delta z$. The outer boundary of the computational domain is at $r=r_{d}$. The inner boundary is at $r=2 \Delta r$, i.e. two grid intervals from the coordinate singularity at the rotation axis. Because of the directionality of operators like $\boldsymbol{u} \cdot \nabla$ and $\boldsymbol{B} \cdot \nabla$, the spatial derivatives are calculated using two-point, one-sided (first-order) finite differences whose directions are chosen to ensure numerical stability at the grid scale. For reasons of symmetry and good behaviour near the coordinate singularity, the finite differencing is done by locally 
approximating the fields $\Psi / r, u_{\phi} / r, \omega_{\phi} / r, A / r, B_{\phi} / r, \vartheta$ and $\mu$ as functions that are linear in $z$ and in $r^{2}$ over a single grid interval. This ensures that the error is $O(\Delta r)$ even for small $r$. Field values for $r<2 \Delta r$ are obtained by extrapolation from $r=3 \Delta r$ and $r=2 \Delta r$, again assuming linear functional dependence on $r^{2}$.

With the parameter values given in table 1 , the dimensionless helium-sublayer and Ekman-layer thicknesses are $\delta_{\chi} / \delta=(\chi / \eta)^{1 / 2} \approx 0.14$ and $\delta_{E k} / \delta=E k^{1 / 2} \approx 0.01$ respectively. We have chosen a vertical grid interval $\Delta z=0.01$, dimensionally $0.01 \delta$, which is small enough to resolve the helium sublayer accurately. This $\Delta z$ is too large to resolve any Ekman layers. However, Ekman layers are prevented from becoming significant by careful choice of the code representing the boundary conditions. By allowing slip velocities and making viscous stresses negligible at the boundaries, we have been able to keep Ekman-layer formation so weak as to play no significant role in the dynamics. Uniform rotation is imposed at and just beneath the bottom of the domain, via the $\boldsymbol{B} \cdot \nabla$ term in the azimuthal component of the induction equation (B 7).

An explicit Eulerian timestepping scheme is used to evolve the system. The time step $\Delta t$ must be small enough to resolve thermal diffusion at the grid scale (which is the fastest process at this scale and therefore determines the Courant-Friedrichs-Lewy condition). So from table $1, \Delta t \lesssim(\eta / \kappa)(\Delta z)^{2}=10^{-2} \times(0.01)^{2}=10^{-6}$, dimensionally $10^{-6} \delta / U$ or $10^{-4}\left(2 \Omega_{i}\right)^{-1}$.

We use a semi-analytical confinement-layer solution, of the kind described in $\S 4$, as the initial condition. The system typically takes several domain-scale magnetic diffusion times to reach a steady state. As explained in $\S 8$, multiple iterations of the peripheral $B_{\phi}(z)$ profile are then required to achieve a steady state with vanishing $B_{\phi}(r)$ at the bottom. To make the computation feasible, in a domain wide enough to accommodate noticeable tilting of the stratification surfaces, we have used $r_{d}=5$, dimensionally $5 \delta$, and a horizontal grid interval $\Delta r=0.1$, dimensionally $0.1 \delta$, larger than the vertical grid interval $\Delta z$ by a factor of 10 . For numerical stability, the dimensionless horizontal viscosity $v_{H}$ and helium diffusivity $\chi_{H}$ must then be chosen so that the diffusive terms in (B 5), (B 6) and (B 10) dominate the advective terms at the gridscale. In practice, we found it sufficient to take $v_{H}=\chi_{H}=10$, i.e. to make them a factor 10 greater than the corresponding vertical diffusivities, as suggested by advective-diffusive scaling when $\Delta r / \Delta z=10$. We have verified, in smaller computational domains, that the coarser horizontal resolution and anisotropic diffusivities do not qualitatively affect the steady state of the system.

At each time step, the azimuthal vorticity $\omega_{\phi}$ is updated and the streamfunction $\Psi$ then computed from (B 3) by inverting the operator $\nabla^{2}-r^{-2}$, approximated using centred differences. The inversion is performed iteratively, using a successiveoverrelaxation method described in Press et al. (1986). During the early evolution, when the dynamics is dominated by time scales not much longer than the time step $\Delta t$, many such iterations are required, at each time step, to achieve convergence. At later times the same degree of convergence can be achieved with far fewer iterations. Since we are interested only in the ultimate steady state, we can tolerate a larger error in the inversion during the system's transient evolution. Further details of the numerical code are spelt out in Wood (2010).

As anticipated from the small values of $R o$ and $E k$, the steady state is found to be close to magnetostrophic balance: the motion is scarcely distinguishable from one in which the balance conditions (3.14) and (3.15) hold exactly. Indeed, in (3.15) the terms in $\alpha_{\vartheta}$ and $\alpha_{\mu}$ dominate so strongly that, in the case of figure 2 for instance, the tilting is barely visible, as has been verified from plots, not shown, of the thermal as 


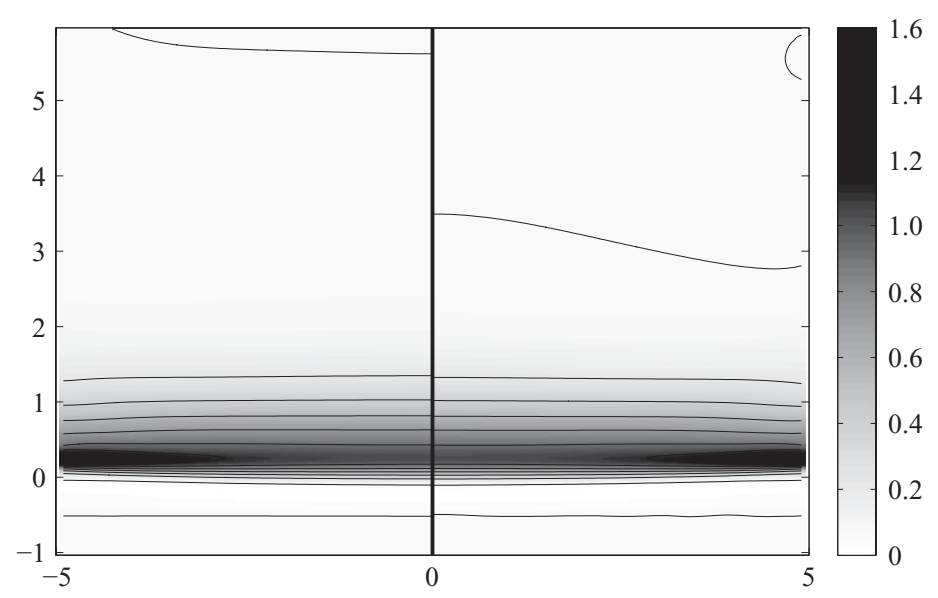

Figure 10. The left and right panels show contours of the left-hand side and right-hand side of (B 11) respectively, in a vertical cross-section for the numerical solution of figures 2,5 , and 6 . The bulk of the confinement layer is close to magnetostrophic balance. The largest fractional departures from balance occur in the regions above and below the confinement layer where both sides of (B 11) are numerically small. Unlike that in figure 2 , the cross-sections shown here span the entire numerical domain including the top part $5 \leqslant z \leqslant 6$.

well as the compositional stratification surfaces. This of course is no more than was anticipated from the scaling arguments of $\S \S 5$ and 6. So the balance (3.15) holds in an almost trivial sense, with the remaining terms scarcely able to produce any noticeable effect. The azimuthal momentum balance described by (3.14) is less trivial, but here also we find that the left-hand side and right-hand side are close to being equal, in the present notation

$$
-\frac{1}{r} \frac{\partial \Psi}{\partial z} \approx \frac{1}{r^{2}} \boldsymbol{B} \cdot \nabla\left(r B_{\phi}\right)
$$

Figure 10 shows the left-hand side and right-hand side of (B 11) for the case of figure 2. The balance is quite accurate despite the modest $R o$ value chosen for the numerical solution, $10^{-2}$ in table 1 .

It might be thought that imposing magnetostrophic balance from the start, as first suggested by Taylor (1963), would filter out all the fast oscillations - including inertial or epicyclic oscillations as well as Alfvén waves, gravity waves, and the various hybrid types - and thereby allow larger time steps to be used. However, the imposition of magnetostrophic balance leads to pathological behaviour at small scales (Walker, Barenghi \& Jones 1998). Far from eliminating or slowing the fast oscillations, the imposition of balance exacerbates the problem, for reasons explained in Appendix C.

\section{Appendix C. Magnetostrophic balance and numerical ill-conditionedness}

Filtering out fast oscillations by imposing some kind of balance is a familiar, and often effective, device in many other problems involving stiff differential equations. A well-known example is that of fluid flow in non-MHD fluid systems with strong rotation (small $\mathrm{Ro}$ ) and stable stratification. The standard 'quasi-geostrophic equations' result from imposing geostrophic or Coriolis-pressure-gradient as well as hydrostatic balance, thereby filtering out inertia and gravity waves as well as sound 
waves. The filtering saves computational resources by allowing relatively large time steps.

Such filtering turns out, however, to be ineffective in the confinement-layer problem. Indeed - at first sight paradoxically - the imposition of magnetostrophic balance exacerbates the timestepping problem. Far from eliminating fast oscillations, it introduces spurious modes of oscillation that are even faster, as shown by Walker et al. (1998) in the context of the terrestrial dynamo problem. Following Walker et al., we show how this pathology can be understood through an idealized analysis of the fast oscillations, first in the unfiltered and then in the filtered equations.

The reason for the pathology is the interplay between the Coriolis and Lorentz forces. Stratification $N^{2}$ is relatively unimportant, as will be shown shortly. We therefore start with the linear theory of MC (magneto-Coriolis) waves, i.e. small plane-wave disturbances to an unstratified, incompressible fluid with solid rotation $\boldsymbol{\Omega}$ and a uniform magnetic field $\boldsymbol{B}$. Neglecting viscosity and magnetic diffusivity, we find the well-known dispersion relation

$$
\omega^{2}-(\boldsymbol{B} \cdot \boldsymbol{k})^{2}= \pm 2 \boldsymbol{\Omega} \cdot \boldsymbol{k} \omega /|\boldsymbol{k}|,
$$

where $\omega$ is the frequency and $\boldsymbol{k}$ is the wavevector, both dimensional here. If we take the limit of rapid rotation, $|\boldsymbol{\Omega}| \rightarrow \infty$, then for most choices of $\boldsymbol{k}$ the four roots of this dispersion relation are asymptotically

and

$$
\begin{aligned}
& \omega \sim \pm \frac{2 \boldsymbol{\Omega} \cdot \boldsymbol{k}}{|\boldsymbol{k}|} \\
& \omega \sim \pm \frac{(\boldsymbol{B} \cdot \boldsymbol{k})^{2}}{2 \boldsymbol{\Omega} \cdot \boldsymbol{k}}|\boldsymbol{k}| .
\end{aligned}
$$

The modes corresponding to $(\mathrm{C} 2)$ are inertial or epicyclic waves - in this context sometimes called 'fast MC waves' - and those corresponding to (C 3) are 'slow MC waves'. By imposing magnetostrophic balance we neglect relative fluid accelerations, which corresponds to dropping the $\omega^{2}$ term from the left-hand side of (C1). The dispersion relation then becomes

$$
\omega= \pm \frac{(\boldsymbol{B} \cdot \boldsymbol{k})^{2}}{2 \boldsymbol{\Omega} \cdot \boldsymbol{k}}|\boldsymbol{k}| .
$$

So imposing magnetostrophic balance eliminates the two 'fast' branches $(\mathrm{C} 2)$ of the full dispersion relation $(\mathrm{C} 1)$.

However, not all modes of the full dispersion relation (C1) have the asymptotic behaviour described by (C2) and (C 3). Even in the presence of rapid rotation, there are always some modes whose $\boldsymbol{k}$ values satisfy

$$
|\boldsymbol{B} \cdot \boldsymbol{k}| \gg|2 \boldsymbol{\Omega} \cdot \boldsymbol{k}| /|\boldsymbol{k}|
$$

by an arbitrarily large factor. For instance we can fix the direction of $\boldsymbol{k}$ and make $|\boldsymbol{k}|$ arbitrarily large. Such modes behave like Alfvén waves, with $\omega \approx \pm \boldsymbol{B} \cdot \boldsymbol{k}$. Imposing magnetostrophic balance removes the mechanism for Alfvén wave propagation, and must therefore alter the behaviour of these modes. In fact their frequencies become arbitrarily higher than Alfvén wave frequencies. This can be seen at once by inspection of (C 4) and (C 5). In summary, even in a rapidly rotating system some modes of the full 
dispersion relation always feel the Lorentz force more strongly than the Coriolis force, i.e., they satisfy (C 5), and these modes become ill-behaved under the assumption of magnetostrophic balance. A numerical scheme that imposes magnetostrophic balance while retaining realistic (Laplacian) magnetic dissipation will therefore be ill-conditioned.

If we introduce stratification $N^{2}$ then (C4) becomes

$$
\omega= \pm \frac{\boldsymbol{B} \cdot \boldsymbol{k}}{2 \boldsymbol{\Omega} \cdot \boldsymbol{k}}\left[(\boldsymbol{B} \cdot \boldsymbol{k})^{2}|\boldsymbol{k}|^{2}+N^{2}\left|\boldsymbol{k}_{H}\right|^{2}\right]^{1 / 2},
$$

where $\boldsymbol{k}_{H}$ is the horizontal projection of $\boldsymbol{k}$. Therefore the stratification (a) makes little difference to the large- $|\boldsymbol{k}|$ behaviour but $(b)$ always increases the frequency of the ill-behaved modes and thereby, if anything, further exacerbates the problem.

\section{REFERENCES}

Braithwaite, J. \& Spruit, H. C. 2004 A fossil origin for the magnetic field in A stars and white dwarfs. Nature 431, 819-821.

Brun, A. S. \& ZAHN, J.-P. 2006 Magnetic confinement of the solar tachocline. Astron. Astrophys. 457, 665-674.

Charbonneau, P. \& MacGregor, K. B. 1993 Angular Momentum Transport in Magnetized Stellar Radiative Zones. II. The Solar Spin-down. Astrophys. J. 417, 762-780.

Charbonnel, C. \& Talon, S. 2007 On Internal Gravity Waves in Low-mass Stars. In Unsolved Problems in Stellar Physics: A Conference in Honor of Douglas Gough (ed. R. J. Stancliffe, J. Dewi, G. Houdek, R. G. Martin \& C. A. Tout), American Institute of Physics Conference Series, vol. 948, pp. 15-26. American Institute of Physics.

Christensen-Dalsganid, J., Gough, D. O. \& Thompson, M. J. 1992 On the rate of destruction of lithium in late-type main-sequence stars. Astron. Astrophys. 264, 518-528.

Christensen-Dalsgand, J., Proffitt, C. R. \& Thompson, M. J. 1993 Effects of diffusion on solar models and their oscillation frequencies. Astrophys. J. 403, L75-L78.

Christensen-DalsgaARd, J. \& Thompson, M. J. 2007 Observational results and issues concerning the tachocline. In The Solar Tachocline (ed. D. W. Hughes, R. Rosner \& N. O. Weiss), p. 53. Cambridge University Press.

Ciacio, F., Degl'Innocenti, S. \& Ricci, B. 1997 Updating standard solar models. Astron. Astrophys. Suppl. 123, 449-454.

DeBnath, L. 1973 On Ekman and Hartmann boundary layers in a rotating fluid. Acta Mechanica 18, 333-341.

Elliott, J. R. 1997 Aspects of the solar tachocline. Astron. Astrophys. 327, 1222-1229.

Elliott, J. R. \& Gough, D. O. 1999 Calibration of the thickness of the solar tachocline. Astrophys. J. 516, 475-481.

Ferraro, V. C. A. 1937 The non-uniform rotation of the Sun and its magnetic field. Mon. Not. R. Astron. Soc. 97, 458-472.

Garaud, P. \& Brummell, N. H. 2008 On the penetration of meridional circulation below the solar convection zone. Astrophys. J. 674, 498-510.

Garaud, P. \& Garaud, J.-D. 2008 Dynamics of the solar tachocline. II. The stratified case. Mon. Not. R. Astron. Soc. 391, 1239-1258.

Gilman, P. A. \& Cally, P. S. 2007 Global MHD instabilities of the tachocline. In The Solar Tachocline (ed. D. W. Hughes, R. Rosner \& N. O. Weiss), p. 243. Cambridge University Press.

Gough, D. 2007 An introduction to the solar tachocline. In The Solar Tachocline (ed. D. W. Hughes, R. Rosner \& N. O. Weiss), p. 3. Cambridge University Press.

Gough, D. O. \& McIntyre, M. E. 1998 Inevitability of a magnetic field in the Sun's radiative interior. Nature 394, 755-757.

Haynes, P. H., McIntyre, M. E., Shepherd, T. G., Marks, C. J. \& Shine, K. P. 1991 On the 'downward control' of extratropical diabatic circulations by eddy-induced mean zonal forces. J. Atmos. Sci. 48, 651-680.

Hughes, D. W., Rosner, R. \& Weiss, N. O. 2007 The Solar Tachocline. Cambridge University Press. 
Kitchatinov, L. L. \& RÜDiger, G. 2006 Magnetic field confinement by meridional flow and the solar tachocline. Astron. Astrophys. 453, 329-333.

Kitchatinov, L. L. \& RÜDiger, G. 2008 Diamagnetic pumping near the base of a stellar convection zone. Astron. Nachr. 329, 372-375.

Kleeorin, N., Rogachevskit, I., Ruzmaikin, A., Soward, A. M. \& Starchenko, S. 1997 Axisymmetric flow between differentially rotating spheres in a dipole magnetic field. J. Fluid Mech. 344, 213-244.

MacGregor, K. B. \& Charbonneau, P. 1999 Angular momentum transport in magnetized stellar radiative zones. IV. Ferraro's theorem and the solar tachocline. Astrophys. J. 519, 911-917.

MCINTYRe, M. 1994 The quasi-biennial oscillation (QBO): some points about the terrestrial QBO and the possibility of related phenomena in the solar interior. In The Solar Engine and Its Influence on Terrestrial Atmosphere and Climate (ed. E. Nesme-Ribes), p. 293. Available at: www.atm.damtp.cam.ac.uk/people/mem/tachocline.

MCINTYRe, M. E. 2003 Solar tachocline dynamics: eddy viscosity, anti-friction, or something in between? In Stellar Astrophysical Fluid Dynamics (ed. M. J. Thompson \& J. ChristensenDalsgaard), pp. 111-130. Cambridge University Press.

McIntyre, M. E. 2007 Magnetic confinement and the sharp tachopause. In The Solar Tachocline (ed. D. W. Hughes, R. Rosner \& N. O. Weiss), p. 183. Cambridge University Press, corrected version available at: www.atm.damtp.cam.ac.uk/people $/ \mathrm{mem} /$ tachocline.

Mestel, L. 1953 Rotation and stellar evolution. Mon. Not. R. Astron. Soc. 113, 716-745.

Mestel, L. 2011 Stellar Magnetism, 2nd edn. Oxford University Press.

Mestel, L. \& Moss, D. L. 1986 On mixing by the Eddington-Sweet circulation. Mon. Not. R. Astron. Soc. 221, 25-51.

Mestel, L. \& Weiss, N. O. 1987 Magnetic fields and non-uniform rotation in stellar radiative zones. Mon. Not. R. Astron. Soc. 226, 123-135.

Parfrey, K. P. \& Menou, K. 2007 The origin of solar activity in the tachocline. Astrophys. J. 667, L207-L210.

Plumb, R. A. \& McEwan, A. D. 1978 The instability of a forced standing wave in a viscous stratified fluid: a laboratory analogue of the quasi-biennial oscillation. J. Atmos. Sci. 35, 1827-1839.

Press, W. H., Flannery, B. P., Teukolsky, S. A. \& Vetterling, W. T. 1986 Numerical Recipes: The Art of Scientific Computing. Cambridge University Press.

Rogers, T. M. \& Glatzmaier, G. A. 2006 Angular Momentum Transport by Gravity Waves in the Solar Interior. Astrophys. J. 653, 756-764.

RÜDIGER, G. \& Kitchatinov, L. L. 1997 The slender solar tachocline: a magnetic model. Astron. Nachr. 318, 273-279.

SchatZMAN, E. 1993 Transport of angular momentum and diffusion by the action of internal waves. Astron. Astrophys. 279, 431-446.

Schou, J., Antia, H. M., Basu, S., Bogart, R. S., Bush, R. I., Chitre, S. M., ChristensenDalsgaard, J., di Mauro, M. P., Dziembowski, W. A., Eff-Darwich, A., Gough, D. O., Haber, D. A., Hoeksema, J. T., Howe, R., Korzennik, S. G., Kosovichev, A. G., Larsen, R. M., Pijpers, F. P., Scherrer, P. H., Sekit, T., Tarbell, T. D., Title, A. M., Thompson, M. J. \& ToOmRe, J. 1998 Helioseismic studies of differential rotation in the solar envelope by the Solar Oscillations Investigation using the Michelson Doppler Imager. Astrophys. J. 505, 390-417.

Spiegel, E. A. \& ZAHn, J.-P. 1992 The solar tachocline. Astron. Astrophys. 265, 106-114.

Spruit, H. C. 2002 Dynamo action by differential rotation in a stably stratified stellar interior. Astron. Astrophys. 381, 923-932.

TAYLOR, J. B. 1963 The magneto-hydrodynamics of a rotating fluid and the earth's dynamo problem. Proc. R. Soc. Lond. A 274, 274-283.

Tobias, S. M., Brummell, N. H., Clune, T. L. \& Toomre, J. 2001 Transport and storage of magnetic field by overshooting turbulent compressible convection. Astrophys. J. 549, 1183-1203.

Vauclair, S., Vauclair, G., Schatzman, E. \& Michaud, G. 1978 Hydrodynamical instabilities in the envelopes of main-sequence stars - Constraints implied by the lithium, beryllium, and boron observations. Astrophys. J. 223, 567-582.

Walker, M. R., Barenghi, C. F. \& Jones, C. A. 1998 A note on dynamo action at asymptotically small Ekman number. Geophys. Astrophys. Fluid Dyn. 88, 261-275. 
Weiss, N. O., Thomas, J. H., Brummell, N. H. \& Tobias, S. M. 2004 The origin of penumbral structure in sunspots: downward pumping of magnetic flux. Astrophys. J. 600, 1073-1090.

WooD, T. S. 2010 The solar tachocline: a self-consistent model of magnetic confinement. PhD thesis, University Library, Superintendent of Manuscripts, Cambridge.

Wood, T. S. \& MCInTYRe, M. E. 2007 Confinement of the Sun's interior magnetic field: some exact boundary-layer solutions. In Unsolved Problems in Stellar Physics: A Conference in Honor of Douglas Gough (ed. R. J. Stancliffe, J. Dewi, G. Houdek, R. G. Martin \& C. A. Tout), American Institute of Physics Conference Series, vol. 948, pp. 303-308. Corrigendum at: www.atm.damtp.cam.ac.uk/people/mem/tachocline. American Institute of Physics.

Zahn, J.-P., Talon, S. \& Matias, J. 1997 Angular momentum transport by internal waves in the solar interior. Astron. Astrophys. 322, 320-328. 